Draft VERsion April 5, 2021

Typeset using LATEX twocolumn style in AASTeX63

\title{
Deep Observations of CO and Free-Free Emission in Ultraluminous Infrared QSO IRAS F07599+6508
}

\author{
Qing-Hua Tan, ${ }^{1}$ Yu Gao, ${ }^{2,1}$ Emanuele Daddi, ${ }^{3}$ Xiao-Yang Xia, ${ }^{4}$ Cai-Na Hao, ${ }^{4}$ Alain Omont, ${ }^{5}$ And \\ Kotaro Kohno ${ }^{6,7}$ \\ ${ }^{1}$ Purple Mountain Observatory $\&$ Key Laboratory for Radio Astronomy, Chinese Academy of Sciences, 10 Yuanhua Road, Nanjing \\ 210023, People's Republic of China; qhtan@pmo.ac.cn \\ ${ }^{2}$ Department of Astronomy, Xiamen University, 422 Siming South Road, Xiamen 361005, People's Republic of China; yugao@xmu.edu.cn \\ ${ }^{3}$ CEA, Irfu, DAp, AIM, Universitè Paris-Saclay, Universitè de Paris, CNRS, F-91191 Gif-sur-Yvette, France \\ ${ }^{4}$ Tianjin Astrophysics Center, Tianjin Normal University, Tianjin 300387, People's Republic of China \\ ${ }^{5}$ Sorbonne Université, UPMC Université Paris 6 and CNRS, UMR 7095, Institut d'Astrophysique de Paris, 98bis boulevard Arago, 75014 \\ Paris, France \\ ${ }^{6}$ Institute of Astronomy, Graduate School of Science, The University of Tokyo, Osawa, Mitaka, Tokyo 181-0015, Japan \\ ${ }^{7}$ Research Center for the Early Universe, Graduate School of Science, The University of Tokyo, 7-3-1 Hongo, Bunkyo-ku, Tokyo \\ 113-0033, Japan
}

\begin{abstract}
Infrared quasi-stellar objects (IR QSOs) are a rare subpopulation selected from ultraluminous infrared galaxies (ULIRGs) and have been regarded as promising candidates of ULIRG-to-optical QSO transition objects. Here we present NOEMA observations of the $\mathrm{CO}(1-0)$ line and $3 \mathrm{~mm}$ continuum emission in an IR QSO IRAS F07599+6508 at $z=0.1486$, which has many properties in common with Mrk 231. The CO emission is found to be resolved with a major axis of $\sim 6.1 \mathrm{kpc}$ that is larger than the size of $\sim 4.0 \mathrm{kpc}$ derived for $3 \mathrm{~mm}$ continuum. We identify two faint $\mathrm{CO}$ features located at a projected distance of $\sim 11.4$ and $19.1 \mathrm{kpc}$ from the galaxy nucleus, respectively, both of which are found to have counterparts in the optical and radio bands and may have a merger origin. A systematic velocity gradient is found in the $\mathrm{CO}$ main component, suggesting that the bulk of molecular gas is likely rotationally supported. Based on the radio-to-millimeter spectral energy distribution and IR data, we estimate that about $30 \%$ of the flux at $3 \mathrm{~mm}$ arises from free-free emission and infer a free-free-derived star formation rate of $77 M_{\odot} \mathrm{yr}^{-1}$, close to the IR estimate corrected for the AGN contribution. We find a high-velocity $\mathrm{CO}$ emission feature at the velocity range of about -1300 to $-2000 \mathrm{~km} \mathrm{~s}^{-1}$. Additional deep CO observations are needed to confirm the presence of a possible very high-velocity $\mathrm{CO}$ extension of the $\mathrm{OH}$ outflow in this IR QSO.
\end{abstract}

Keywords: galaxies: active - galaxies: evolution - galaxies: ISM - galaxies: starburst - ISM: molecules

\section{INTRODUCTION}

Mergers of gas-rich galaxies may trigger both intense star formation and nuclear activity, leading to a violent transition from an obscured accretion and starburst stage to an optically bright quasistellar object (QSO) and eventually an elliptical galaxy (e.g., Sanders et al. 1988a,b; Barnes \& Hernquist 1992; Di Matteo et al. 2005; Hopkins et al. 2006). In this merger-driven evolutionary scenario, the active galactic nucleus (AGN) was thought to be energetically dominant but obscured in the ultraluminous infrared galaxy (ULIRG; $L_{\mathrm{IR}} \geqslant 10^{12} L_{\odot}$ ) phase, where the most luminous sources that reach a QSO-like luminosity represent the rare ULIRG-to-QSO transition objects. These systems are very likely experiencing the so-called feedback or blowout phase that expels the gas of the host galaxy in the form of outflowing winds, which have been invoked as one of the main drivers for regulating the growth of the stellar mass and black hole $(\mathrm{BH})$ accretion and producing the tight BH-bulge mass relation (see Silk \& Rees 1998; Hopkins et al. 2008; Fabian 2012; Kormendy \& Ho 2013; King \& Pounds 2015, and references therein), and therefore represent a key phase in the lifetime of a quasar.

The large gas masses discovered in local ULIRGs via $\mathrm{CO}$ observations have established the presence of abundant fuel for the intense star formation activity (e.g., Solomon et al. 1997). Along with the clear evidence that many (U)LIRGs are strong dynamical interactions and mergers (Sanders \& Mirabel 1996), all the properties make these systems unique local examples of dustenshrouded galaxies at high- $z$. Xia et al. (2012) have investigated for the first time the CO survey in 19 ultraluminous infrared type 1 QSOs, selected from the local ULIRGs and referred to as IR QSOs (see Zheng et al. 2002). These IR-selected type 1 QSOs (IR QSOs) were 
originally compiled from the ULIRGs in the QDOT redshift survey (Lawrence et al. 1999), the 1 Jy ULIRG survey (Kim \& Sanders 1998), and the cross-correlation of the IRAS Point Source Catalog with the ROSAT Allsky Survey Catalog. Studies of luminosity function for large samples of IRAS galaxies reveal that the space densities of ULIRGs and QSOs are comparable in the local universe (Soifer et al. 1987), while the fraction of IR QSOs is less than $10 \%$ of ULIRGs (Lawrence et al. 1999), indicating that the IR QSO phase may last only a few times $10^{7} \mathrm{yr}$ if the space density of objects is simply related to the timescale of different phases (Hao et al. 2005).

The CO observations of IR QSOs show that the molecular gas masses are a few times $10^{9}-10^{10} M_{\odot}$ (Xia et al. 2012), similar to that found in local ULIRGs but significantly higher than that of optically selected PG QSOs (e.g., Scoville et al. 2003; Evans et al. 2001, 2006; Shangguan et al. 2020). This supports the scenario that IR QSOs are rich of fuel to feed both the star formation and the accretion of AGN. Similar conclusion has also been reached recently for type 2 QSOs, of which a sample of QSO hosts are found to be gas-rich based on the CO observations (Jarvis et al. 2020). Together with the far-IR excess observed in IR QSOs (Hao et al. 2005), and the multi-wavelengths properties (i.e., large blueshifts in optical emission lines, warm IR color, intermediate slope of mid-IR spectral energy distribution (SED) between ULIRGs and PG QSOs, and high accretion rates; Zheng et al. 2002; Cao et al. 2008), all of these properties imply that IR QSOs are likely the objects caught in the short-lived transition phase between ULIRG and QSO stages (Canalizo \& Stockton 2001).

Compared to the local (U)LIRGs that a large number have been spatially resolved in molecular gas emission (e.g., Downes \& Solomon 1998; Wilson et al. 2008; Ueda et al. 2014; Xu et al. 2014, 2015; Imanishi et al. 2019), except Mrk 231, the nearest IR QSO at $z=0.042$ that has been well-studied in both multi-wavelength and multi-scale (e.g., Bryant \& Scoville 1996; Taylor et al. 1999; Fischer et al. 2010; Rupke \& Veilleux 2011; Feruglio et al. 2015; Cicone et al. 2020), until recently the $\mathrm{CO}$ emission have been spatially resolved on subkiloparsec (sub-kpc) scale in a handful of IR QSOs with the ALMA (Tan et al. 2019). It was found that the host galaxies of IR QSOs resolved with ALMA have a diverse variety of $\mathrm{CO}$ morphology and kinematics (i.e., rotating disk, disturbed structure, and multiple interacting sources), indicative of more complicated evolutionary stages from merging (U)LIRGs to QSOs. This is somewhat unexpected since the objects in IR QSO phase are typically characterized by the final coalescence of the galaxies, according to the models of mergerdriven evolutionary scenario (Barnes \& Hernquist 1992; Hopkins et al. 2008). However, such a classification suffers from small number statistics, spatially resolved $\mathrm{CO}$ observations in more IR-luminous QSOs are required for a meaningful statistical study.

In this paper, we present $\mathrm{CO}(1-0)$ imaging of IRAS F07599+6508 obtained with the IRAM NOrthern Extended Millimeter Array (NOEMA) interferometer. IRAS F07599+6508 is an IR QSO at $z=0.149$ with a total IR luminosity of $3.5 \times 10^{12} L_{\odot}$ and AGN bolometric luminosity of $L_{\mathrm{AGN}, \text { bol }}=3.9 \times 10^{12} L_{\odot}$ (Hao et al. 2005). It is an active galaxy of particular interest because of its highly similarity to Mrk 231, the closest and most prominent template of local IR QSOs, but at a higher redshift and lack of detailed studies on the cold molecular gas of the host galaxy, especially the spatial distribution and kinematics. Similar to Mrk 231, IRAS F07599+6508 is a low-ionization broad absorption-line (BAL) QSO with strong FeII emission (e.g., Kwan et al. 1995; Véron-Cetty et al. 2006), which is a very rare subclass of BAL QSOs that may represent young, heavily enshrouded AGNs, where the cocoon of gas and dust has a large covering factor (Boroson \& Meyers 1992; Zheng et al. 2002).

The IRAM $30 \mathrm{~m}$ CO observations show massive amounts of molecular gas residing in IRAS F07599+6508 and a broad FWHM line width of $380 \pm 14 \mathrm{~km} \mathrm{~s}^{-1}$ and $490 \pm 40 \mathrm{~km} \mathrm{~s}^{-1}$ for the $\mathrm{CO}(1-0)$ and $\mathrm{CO}(2-1)$ line, respectively (Xia et al. 2012). Based on six independent methods, Veilleux et al. (2009) estimate an average fraction of AGN contribution to the bolometric luminosity of $\alpha_{\mathrm{AGN}}=0.75$, consistent with other IR QSOs (e.g., $\sim 70 \%$ for Mrk 231) where the $\alpha_{\mathrm{AGN}}$ is found to be typically higher than that $(\sim 35 \%-40 \%)$ of local cooler ULIRGs. The weakness of both soft $\left(L_{0.1-2.4 \mathrm{keV}}=6.9 \times\right.$ $\left.10^{42} \mathrm{erg} \mathrm{s}^{-1}\right)$ and hard X-ray emission $\left(L_{2-10 \mathrm{keV}}=\right.$ $1.3 \times 10^{42} \mathrm{erg} \mathrm{s}^{-1}$ ) seen in IRAS F07599+6508 (e.g., Xia et al. 2001; Brightman \& Nandra 2011; Luo et al. 2014; La Caria et al. 2019), i.e., the X-ray emission is not at the level expected from its optical/UV emission compared to typical QSOs, imply that its central AGN is very likely highly obscured.

The existence of multi-phase outflow winds in IRAS F07599+6508 has been demonstrated by the detection of large blueshifts $\left(\sim 2000 \mathrm{~km} \mathrm{~s}^{-1}\right)$ of optical emission lines, Na I D interstellar absorption line, and OH $119 \mu \mathrm{m}$ P-Cygni/blue-shifted absorption profile, closely resemble to Mrk 231 (Boroson \& Meyers 1992; Zheng et al. 2002; Spoon et al. 2013; Veilleux et al. 2013; Rupke et al. 2017). The sensitive $\mathrm{CO}(1-0)$ mapping observations will help constrain the distribution and kinematics of the molecular gas reservoir in IRAS F07599+6508 and offer the possibility of detecting the molecular outflow based on the presence of broad wings in the $\mathrm{CO}$ line emission. Tremendous amount of observing time were devoted to Mrk 231 on NOEMA revealing the outstanding molecular outflows including in dense gas tracers (Aalto et al. 2012), and likely the ten- 
tative detection of large-scale molecular oxygen emission (Wang et al. 2020).

Alike to Mrk 231, IRAS F07599+6508 has typical features expected for a system transiting from the heavily obscured starburst phase to the unobscured QSO phase, and could also be regarded as a representative of IR QSOs which have low number density in the local universe but are expected to be common at high redshift (Veilleux et al. 1999; Le Floc'h et al. 2005), and thus provides nearby laboratory to probe the ULIRG-tooptical QSO transition phase that may hold the key to understanding the galaxy evolution in a merger-driven scenario.

The paper is organized as follows. In Section 2, we describe the NOEMA observations and data reduction process. Section 3 presents the results from the analysis of $3 \mathrm{~mm}$ continuum and $\mathrm{CO}(1-0)$ line data, including morphology, spatially decomposed CO components, kinematics, and an analysis of archival radio continuum data from VLA. In section 4, we discuss the nature of $3 \mathrm{~mm}$ continuum emission, the origin of the offcenter CO components, and make a comparison of the molecular gas properties between IRAS F07599+6508 and Mrk 231. We summarize our main findings in Section 5. Throughout this work, we assume a standard $\Lambda \mathrm{CDM}$ cosmology with $H_{0}=70 \mathrm{~km} \mathrm{~s}^{-1} \mathrm{Mpc}^{-1}, \Omega_{\mathrm{m}}=$ 0.3 , and $\Omega_{\Lambda}=0.7$.

\section{OBSERVATIONS AND DATA REDUCTION}

\subsection{NOEMA CO(1-0) Observations}

We have observed the $\mathrm{CO}(1-0)$ line and the continuum emission in IRAS F07599+6508 with the IRAM NOEMA interferometer between May and July 2018. The observations were obtained with a total on-source time of $12.7 \mathrm{~h}$ in the compact array D-configuration (with eight antennas covering baselines of 15.2 - 175.9 $\mathrm{m})$. We used the PolyFix correlator in $3 \mathrm{~mm}$ Band 1, which provides $2 \times 8 \mathrm{GHz}$ of instantaneous dualpolarisation bandwidth with a spectral resolution of $2 \mathrm{MHz}$ (corresponding to $6.0 \mathrm{~km} \mathrm{~s}^{-1}$ at the redshifted $\mathrm{CO}(1-0)$ frequency of $100.353 \mathrm{GHz})$. The tuning frequency was set to $98.2 \mathrm{GHz}$ with frequency ranged from 94.2 to $102.2 \mathrm{GHz}$ in the Upper SideBand (USB) and 78.8 to $86.8 \mathrm{GHz}$ in the Lower SideBand (LSB). In this paper, we analyze the $\mathrm{CO}(1-0)$ line and the continuum data in the USB; the rest of detected lines (i.e., $\mathrm{CN}(1-0)$ lines at 113.191 and $113.491 \mathrm{GHz}, \mathrm{CO}(1-0)$ isotopologue lines, and the $109.174 \mathrm{GHz} \mathrm{HC}_{3} \mathrm{~N}$ (12-11) line) covered in the USB and the LSB line data will be presented in a future paper.

The data calibration was performed using the GILDAS $^{1}$ package CLIC with help from the staff in Grenoble. The typical uncertainty of flux calibration

${ }^{1}$ http://iram.fr/IRAMFR/GILDAS; for more information, see Guilloteau \& Lucas (2000) in the $3 \mathrm{~mm}$ band is about $10 \%$. We binned the $u v$ tables by $20 \mathrm{~km} \mathrm{~s}^{-1}$ and redefined the rest frequency to the sky frequency of $\mathrm{CO}(1-0)$ transition. We then used the software GILDAS/MAPPING for image cleaning and analysis in the $u v$-plane. We also exported the data to a $\mathrm{CASA}^{2}$-compatible format for a comparison analysis. The continuum $u v$ table was created from the calibrated visibilities by averaging the line-free channels using the MAPPING task uv_continuum with line emission channels filtered (uv_filter task). The continuum emission was subtracted from the line $u v$-data by fitting a first-order polynomial baseline for each visibility (uv_baseline task). The USB and LSB continuum uvdata were combined using the uv_merge task to form a single output table centered at $90.5 \mathrm{GHz}$. The synthesized beam size using natural weighting is $5 .{ }^{\prime \prime} 67 \times 3 .{ }^{\prime \prime} 81$ and the NOEMA primary beam at the sky frequency of $\mathrm{CO}(1-0)$ is $50 .{ }^{\prime \prime} 2 \times 50 .{ }^{\prime \prime} 2$. We obtain a sensitivity of $\sim 0.44 \mathrm{mJy}^{-1}$ beam $^{-1}$ in the spectral velocity resolution of $20 \mathrm{~km} \mathrm{~s}^{-1}$ and $\sim 10 \mu \mathrm{Jy}$ beam $^{-1}$ for the continuum image.

\subsection{Archival VLA Radio Data}

In this work we also make use of ancillary radio continuum data observed with the Very Large Array (VLA) for a comprehensive analysis. IRAS F07599+6508 has been observed at 1.49, 4.86, 8.44, 14.94, and $22.46 \mathrm{GHz}$ in CD configuration (project ID: AB0783). Higher resolution observations in $\mathrm{AB}$ configuration (project ID: AH0406) were available at $1.49,4.86$, and $8.44 \mathrm{GH}$, as well as in A configuration at $14.94 \mathrm{GHz}$ (project ID: AN0104). We obtained the raw data sets from the VLA archive ${ }^{3}$. The data flagging and reduction were performed by using the AIPS package (van Moorsel et al. 1996). The full image at each frequency and array was processed individually with the AIPS CLEAN task imagr and then transferred to FITS format for further analysis with the CASA package. The synthesized beam using Briggs weighting with a robustness parameter of 0.5 ranges from $65 .{ }^{\prime \prime} 2 \times 28 .{ }^{\prime \prime} 3$ at $1.49 \mathrm{GHz}$ to $1 .^{\prime \prime} 67 \times 1 .{ }^{\prime \prime} 30$ at $43.34 \mathrm{GHz}$ for observations in CD configuration, and from $5 .{ }^{\prime \prime} 33 \times 2 .{ }^{\prime \prime} 08$ at 1.49 $\mathrm{GHz}$ to $0 .{ }^{\prime \prime} 18 \times 0 .{ }^{\prime \prime} 13$ at $14.94 \mathrm{GHz}$ for higher resolution observations in $\mathrm{AB}$ and $\mathrm{A}$ configurations.

\section{RESULTS AND ANALYSIS}

\section{1. $3 \mathrm{~mm}$ Continuum Emission}

The continuum emission at $90.5 \mathrm{GHz}$ (rest-frame 103.9 $\mathrm{GHz} ; 3 \mathrm{~mm}$ ) is clearly detected towards the nucleus of IRAS F07599+6508. Figure 1 shows the $3 \mathrm{~mm}$ continuum map and the corresponding $u v$-plot that shows the amplitude of the visibilities as a function of the $u v$ distance. It can be seen from the $u v$-data that the $3 \mathrm{~mm}$

\footnotetext{
${ }^{2}$ http://casa.nrao.edu; see McMullin et al. (2007)

${ }^{3}$ https://science.nrao.edu/facilities/vla/archive/index
} 

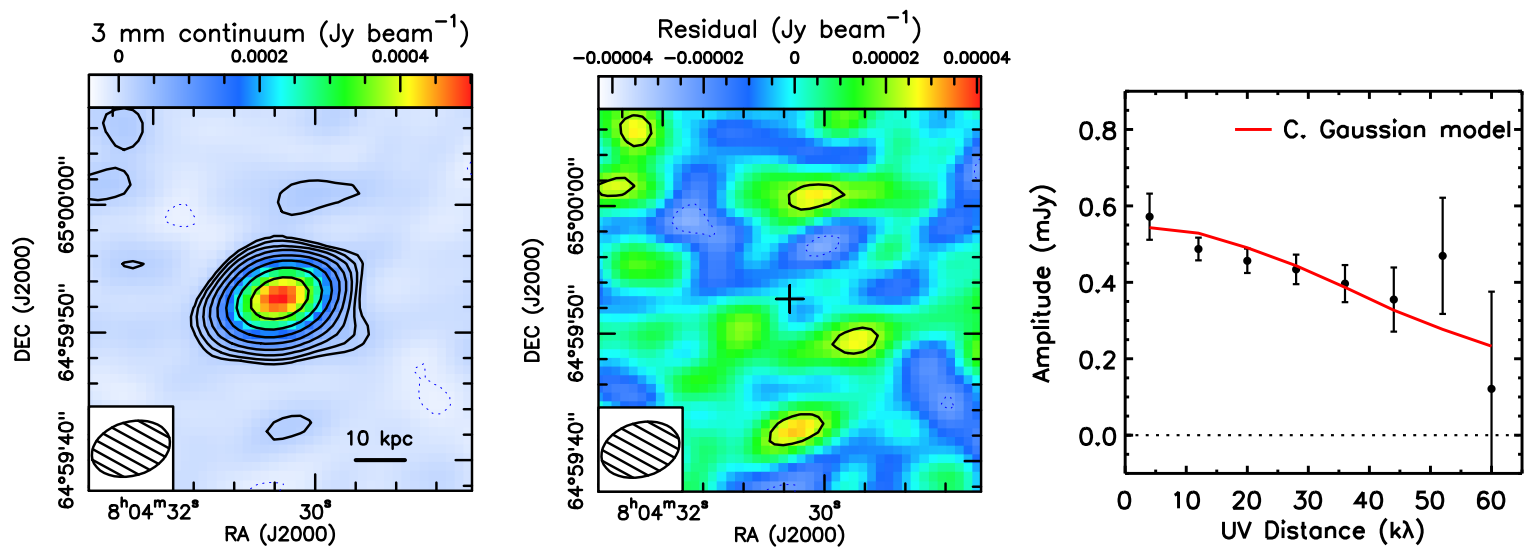

Figure 1. NOEMA cleaned map of $3 \mathrm{~mm}$ continuum emission (left), the residual map of $3 \mathrm{~mm}$ emission after subtracting a circular Gaussian model (middle), and the corresponding $u v$-plot showing the visibility amplitude as a function of the $u v$ distance (right) for IRAS F07599+6508. For $3 \mathrm{~mm}$ continuum map, contours start at $\pm 2 \sigma\left(1 \sigma \mathrm{rms}^{\text {noise is } 10 \mu \mathrm{Jy} \text { beam }}{ }^{-1}\right)$ and increase by a factor of 1.5, with positive (negative) contours shown as solid (dotted) lines, while for the residual map, contours start at $\pm 2 \sigma$ and increase in steps of $1 \sigma$. The synthesized beam of $6 .^{\prime \prime} 25 \times 4 .^{\prime \prime} 22$ with position angle $106.2^{\circ}$ is shown in the bottom left corner. The plus sign indicates the centroid of the $3 \mathrm{~mm}$ continuum emission derived from a circular Gaussian fit to the $u v$-data. In the right panel, the black circles with error bars shown in the $u v$-plot are the data binned in $u v$-radius steps of $8 \mathrm{k} \lambda$. Error bars show the statistical photon noise on the average amplitudein each bin. The red curve indicates the best-fitting model with a circular Gaussian function to the $u v$-data.

continuum source is resolved in our observations, as an unresolved source would give a flat distribution of visibilities.

Assuming that the surface brightness distribution of the continuum follow a symmetric Gaussian profile, we performed a fit to the visibilities in GILDAS using task uv_fit and found that the continuum source is best fitted by a two-dimensional (2D) circular Gaussian model with a deconvolved full width at half maximum (FWHM) size of $1 .{ }^{\prime \prime} 53 \pm 0 .{ }^{\prime \prime} 19(\sim 4.0 \pm$ $0.5 \mathrm{kpc}$ ) and a flux density of $560 \pm 20 \mu \mathrm{Jy}$ (see Table 1). A 2D elliptical Gaussian model fit to the continuum visibilities returns a deconvolved FWHM size of $\left(1 .^{\prime \prime} 54 \pm 0 .{ }^{\prime \prime} 22\right) \times\left(1 .^{\prime \prime} 50 \pm 0 .{ }^{\prime \prime} 39\right)$ and a flux density of $560 \pm 20 \mu \mathrm{Jy}$, which are in good agreement with those derived from fit with a circular Gaussian model, but with slightly larger uncertainties on the size measurement. In addition, the position angle $\left(0^{\circ} \pm 293^{\circ}\right)$ derived from elliptical Gaussian fit is rather uncertain due to the large uncertainty.

In the middle panel of Figure 1, we show the residual after removing the fit with a circular Gaussian model. No significant peaks or negative regions with signal-tonoise ratio $(\mathrm{SNR}) \gtrsim 3$ are found in the residual map. The red curve in $u v$-plot (see the right panel of Figure 1) shows the result of circular Gaussian model fit. Moreover, it can be seen from the right panel of Figure 1 , most data points are consistent with the model fit (see the red curve) with a circular Gaussian function within $1 \sigma$. All of these suggest that a single circular Gaussian model may provide a good description to the $3 \mathrm{~mm}$ continuum emission based on the current data.
As an independent check on the results from our analysis of $u v$-data, we also measured the size and flux of the continuum emission by fitting the data in the image plane using the imfit task in CASA. A 2D Gaussian fit to the $3 \mathrm{~mm}$ continuum map of Figure 1 finds an deconvolved FWHM size of $\left(1 .^{\prime \prime} 84 \pm 0 . .^{\prime \prime} 45\right) \times\left(1 .^{\prime \prime} 40 \pm 0 .^{\prime \prime} 49\right)$ and an integrated flux of $550 \pm 20 \mu \mathrm{Jy}$, consistent with the estimates from the uv_fit within $1 \sigma$ uncertainties.

\section{2. $C O(1-0)$ Line Emission}

\subsubsection{CO morphology and spatial decomposition}

Figure 2 shows the continuum-subtracted $\mathrm{CO}(1-0)$ spectrum and the integrated intensity map of IRAS F07599+6508. The CO spectrum is extracted by fitting a point-source model to the $u v$-table using the uv_fit task. By fitting a Gaussian profile to the spectrum, we measure a redshift of $z_{\mathrm{CO}}=0.1486$, being used to set the systematic velocity. The velocity width (FWHM) derived from the Gaussian fit to the spectrum is $430 \pm 10 \mathrm{~km} \mathrm{~s}^{-1}$. We note that the $\operatorname{CO}(1-0)$ line profile is asymmetrical and a single Gaussian function does not provide a good fit to the line peak and wings. A double-peak profile is detected at the line center albeit not very prominent, which is often taken to indicate orbital motion in a merger or a rotating disk (e.g., Downes \& Solomon 1998; Greve et al. 2005; Daddi et al. 2010). In addition, the line profile also shows a slightly weaker peak at velocity $\sim 100-150$ $\mathrm{km} \mathrm{s}^{-1}$, indicating that the motion of molecular gas in the host galaxy is complex.

The irregular morphology of the $\mathrm{CO}$ emission with extended structure is shown by the map in the right panel of Figure 2, obtained by collapsing the channels in the 

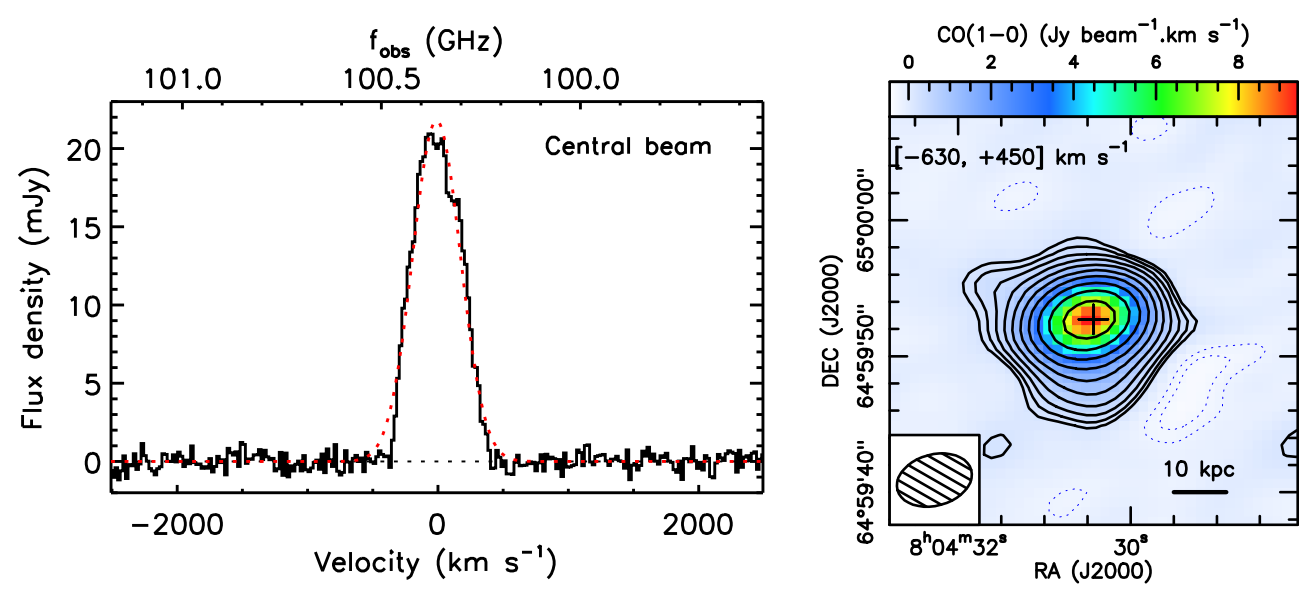

Figure 2. NOEMA continuum-subtracted spectrum (left) and cleaned map (right) of CO(1-0) emission of IRAS F07599+6508. The CO(1-0) spectrum is extracted from the central beam and the spectrum is binned to a channel width of $\sim 20 \mathrm{~km} \mathrm{~s}^{-1}$. The red dotted line represents a Gaussian fit to the spectrum. The zero velocity corresponds to the redshift of $z_{\mathrm{CO}}=0.1486$ derived from a Gaussian fit to the spectrum. The map of $\mathrm{CO}(1-0)$ emission is integrated in the $[-630,+450] \mathrm{km} \mathrm{s}^{-1}$ velocity range. Contours start at $\pm 2 \sigma\left(1 \sigma \mathrm{rms}\right.$ noise is $\left.0.092 \mathrm{Jy}_{\text {beam }}^{-1} \mathrm{~km} \mathrm{~s}^{-1}\right)$ and increase by a factor of 1.5 , with positive (negative) contours shown as solid (dotted) lines. The synthesized beam $\left(5 .{ }^{\prime \prime} 61 \times 3 .{ }^{\prime \prime} 77\right)$ is shown in the bottom left corner of the map. The plus sign indicates the peak of $3 \mathrm{~mm}$ continuum emission.

$[-630,+450] \mathrm{km} \mathrm{s}^{-1}$ velocity range where the flux is positive in each channel when the $\mathrm{CO}$ spectrum is rebinned to a channel width of $\sim 60 \mathrm{~km} \mathrm{~s}^{-1}$. The CO emission appears to be resolved with an extension to the northeast and to the south of the nucleus, respectively. To characterize the distribution of $\mathrm{CO}$ emission, we fitted an elliptical Gaussian model to the $u v$-data with channels averaged in the $[-630,+450] \mathrm{km} \mathrm{s}^{-1}$ velocity range using the task uv_fit. The best fit gives a source size FWHM of $\left(2 .^{\prime \prime} 33 \pm 0 . .^{\prime \prime} 11\right) \times\left(1 .^{\prime \prime} 91 \pm 0 .{ }^{\prime \prime} 07\right)$. The peak of $\mathrm{CO}$ emission is found to be co-spatial with the $3 \mathrm{~mm}$ continuum emission.

In the residual map after subtraction of an elliptical Gaussian component, we detect two off-center CO subcomponents at above $5 \sigma$, which we denote as northeast $(\mathrm{NE})$ and south $(\mathrm{S})$, and we refer to the main component fitted with an elliptical Gaussian in the galaxy center as F07599C (see Figure 3). To take into account the off-center components, we remake the fitting in the $u v$ plane using combinations of functions by allowing all parameters to be free in the modeling, and the details of the model fitting are described in Appendix A. The best fit shows that the $\mathrm{CO}$ emission can be spatially decomposed into three components, an elliptical Gaussian source (F07599C) and two point sources (F07599S and F07599NE).

For the main component F07599C, the model fit returns a source size FWHM of $\left(2 .^{\prime \prime} 35 \pm 0 .^{\prime \prime} 11\right) \times\left(1 . .^{\prime \prime} 66 \pm\right.$ $\left.0 .^{\prime \prime} 09\right)$, corresponding to 6.1 by $4.3 \mathrm{kpc}$, which is about two times of the median source size $(\sim 3.2 \mathrm{kpc})$ measured for the local IR QSOs with well-resolved CO morphology (Tan et al. 2019). The three components identified in the $\mathrm{CO}$ emission and their parameters are listed in Table 1.

\subsubsection{Properties of the decomposed CO components}

Figure 4 shows the $\mathrm{CO}(1-0)$ spectra of the three components and the corresponding velocity-integrated $\mathrm{CO}$ maps. The CO spectra of the three components are extracted simultaneously from the line cube in the $u v$ plane with fitting models identical to the best-fit models summarized in Table 1 (see Section 3.2.1). The spectrum of $\mathrm{F} 07599 \mathrm{C}$ is obtained with $u v$-modeling fit using an elliptical Gaussian function by fixing the parameters of centroid and major/minor axes, while for the offcenter components, F07599S and F07599NE, which are offset from the nucleus of main component with a projected distance of $4 .^{\prime \prime} 4(\sim 11.4 \mathrm{kpc})$ and $7 .^{\prime \prime} 4(\sim 19.1$ $\mathrm{kpc}$ ), respectively, the spectra are extracted by a point source model with centroids fixed.

A single Gaussian fit to the line profile of F07599C and F07599NE shown in Figure 4 yields a peak flux density of $26.0 \pm 0.3 \mathrm{mJy}$ and $1.0 \pm 0.2 \mathrm{mJy}$, and an FWHM of $430 \pm 10 \mathrm{~km} \mathrm{~s}^{-1}$ and $390 \pm 70 \mathrm{~km} \mathrm{~s}^{-1}$, respectively. F07599NE has a velocity offset of $40 \pm 30 \mathrm{~km} \mathrm{~s}^{-1}$, consistent within the uncertainties with the systematic velocity. For F07599S, the spectrum is best fitted by two nested Gaussians (with a shared centre), e.g., a narrow Gaussian to fit the central core of the line, and a broad Gaussian to fit the broad emission. The best-fit gives a peak flux density of $3.9 \pm 0.4 \mathrm{mJy}$ and $1.3 \pm 0.3 \mathrm{mJy}$, and an FWHM of $100 \pm 10 \mathrm{~km} \mathrm{~s}^{-1}$ and $500 \pm 80 \mathrm{~km} \mathrm{~s}^{-1}$ with velocity centroid redshifted at a velocity of about $100 \pm 10 \mathrm{~km} \mathrm{~s}^{-1}$ for the narrow and broad emission, respectively. The results of Gaussian fits derived for the three components are reported in Table 1.

Similar to the CO spectrum extracted from the central beam, the CO line profile of F07599C shows characteristic double peak at the line center and a weaker peak at 

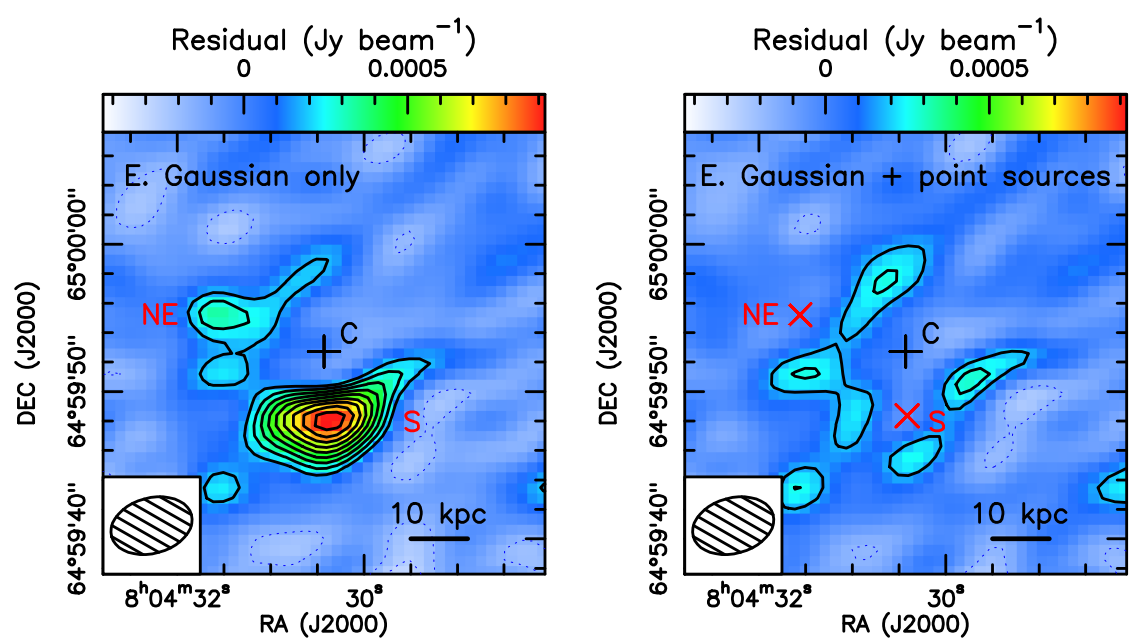

Figure 3. Residual maps of $\mathrm{CO}(1-0)$ emission after subtracting a single elliptical Gaussian model (left) and a combination of an ellipical Gaussian model and two point sources models (right). The black plus sign marks the peak of $3 \mathrm{~mm}$ continuum emission, while the two red crosses indicate the peak positions of the two point-like CO feature. Contours start at $\pm 2 \sigma$ and increase in steps of $1 \sigma$ for both maps. The two point-like subcomponents are labeled as F07599S and F07599NE, respectively.

Table 1. Measured properties of $\mathrm{CO}(1-0)$ line and $3 \mathrm{~mm}$ continuum emission of IRAS F07599+6508

\begin{tabular}{|c|c|c|c|c|c|c|c|}
\hline Emission & Component & $\begin{array}{c}\text { R.A. } \\
\text { (J2000) }\end{array}$ & $\begin{array}{c}\text { Decl. } \\
(\mathrm{J} 2000)\end{array}$ & $\begin{array}{c}\text { Size FWHM } \\
(\operatorname{arcsec})\end{array}$ & $\begin{array}{c}\mathrm{FWHM}_{\mathrm{CO}(1-0)} \\
\left(\mathrm{km} \mathrm{s}^{-1}\right)\end{array}$ & $\begin{array}{c}v_{\text {offset }} \\
\left(\mathrm{km} \mathrm{s}^{-1}\right)\end{array}$ & Integrated Flux \\
\hline (1) & (2) & (3) & (4) & (5) & (6) & (7) & (8) \\
\hline \multirow[t]{3}{*}{$\mathrm{CO}(1-0)$} & F07599C & $08: 04: 30.468 \pm 0.004$ & $64: 59: 52.72 \pm 0.02$ & $(2.35 \pm 0.11) \times(1.66 \pm 0.09)$ & $430 \pm 10$ & 0 & $11.24 \pm 0.09 \mathrm{Jy} \mathrm{km} \mathrm{s}^{-1}$ \\
\hline & F07599S & 08:04:30.41 \pm 0.03 & $64: 59: 48.36 \pm 0.12$ & $\ldots$ & $\begin{array}{c}100 \pm 10 \text { (narrow) } \\
500 \pm 80 \text { (broad) }\end{array}$ & $100 \pm 10$ & $1.12 \pm 0.07 \mathrm{Jy} \mathrm{km} \mathrm{s}{ }^{-1}$ \\
\hline & F07599NE & $08: 04: 31.56 \pm 0.07$ & $64: 59: 55.2 \pm 0.3$ & $\ldots$ & $390 \pm 70$ & $40 \pm 30$ & $0.45 \pm 0.07 \mathrm{Jy} \mathrm{km} \mathrm{s}^{-1}$ \\
\hline
\end{tabular}

Note-Column (1): $\mathrm{CO}(1-0)$ line and $3 \mathrm{~mm}$ continuum emission. Column (2): components of $\mathrm{CO}(1-0)$ emission from source spatial decomposition in the $u v$-plane. Column (3) and (4): source position derived from model fit to the $u v$-data. Column (5): FWHM source size derived from model fitting to the visibilities using the GILDAS task uv_fit. Dots in the column indicate an unresolved component. The physical scale of $1^{\prime \prime}$ corresponds to $\sim 2.6 \mathrm{kpc}$. Column (6): FWHM CO line width derived from a Gaussian profile fit to the spectrum (except for F07599S, where the $\mathrm{CO}$ spectrum is fitted simultaneously with two Gaussians consist of one narrow and one broad components centered at the same velocity). Column(7): $\mathrm{CO}(1-0)$ line centroid velocity offset from the systematic velocity. The centroid velocity is derived from a Gaussian fit to the spectrum. Column (8): $\mathrm{CO}(1-0)$ velocity-integrated intensity and $3 \mathrm{~mm}$ continuum emission flux. The CO integrated fluxes are measured from the spectra shown in Figure 4 by integrating the intensity over the line-emitting region in each channel. The continuum flux is measured from a circular Gaussian fit to the $u v$-data. Errors in fluxes quoted in this table are statistical and do not include the absolute flux calibration uncertainty.

the velocity $\sim 100-150 \mathrm{~km} \mathrm{~s}^{-1}$, which is found to be coincident with the CO line centroid velocity of F07599S (see Figure 4, top panel). The velocity coincidence is likely to suggest that a significant portion of molecular gas are detected in the overlap region between F07599C and F07599S, where the perturbation of molecular gas make an imprint on the CO line profile. Higher spatial resolution observations are needed to resolve the emission and clarify the nature of the source.

The CO morphology of each component is shown by the map in the bottom-right panel of Figure 4, obtained by averaging the velocity range of $[-330,+330] \mathrm{km} \mathrm{s}^{-1}$, $[-270,+330] \mathrm{km} \mathrm{s}^{-1}$, and $[-330,+270] \mathrm{km} \mathrm{s}^{-1}$, where the emission is detected in consecutive channels at above
$1 \sigma$, for F07599C, F07599S, and F07599NE, respectively. The velocity-integrated $\mathrm{CO}$ emission of each component was imaged separately by collapsing the velocity channels over the line-emitting region. It can be seen from Figure 4 that the superimposed image of the three components agrees well with the global CO map that is integrated in the $[-630,+450] \mathrm{km} \mathrm{s}^{-1}$ velocity range with coincident image contours (e.g., contours at a $3 \sigma$ level). We derive an integrated flux density of $11.24 \pm 0.09 \mathrm{Jy}$ $\mathrm{km} \mathrm{s}^{-1}, 1.12 \pm 0.07 \mathrm{Jy} \mathrm{km} \mathrm{s}^{-1}$, and $0.45 \pm 0.07 \mathrm{Jy} \mathrm{km} \mathrm{s}^{-1}$, by integrating the intensity over the line-emitting region in each channel of the spectra shown in Figure 4, for F07599C, F07599S, and F07599NE, respectively. From this analysis, the two subcomponents (F07599S and 

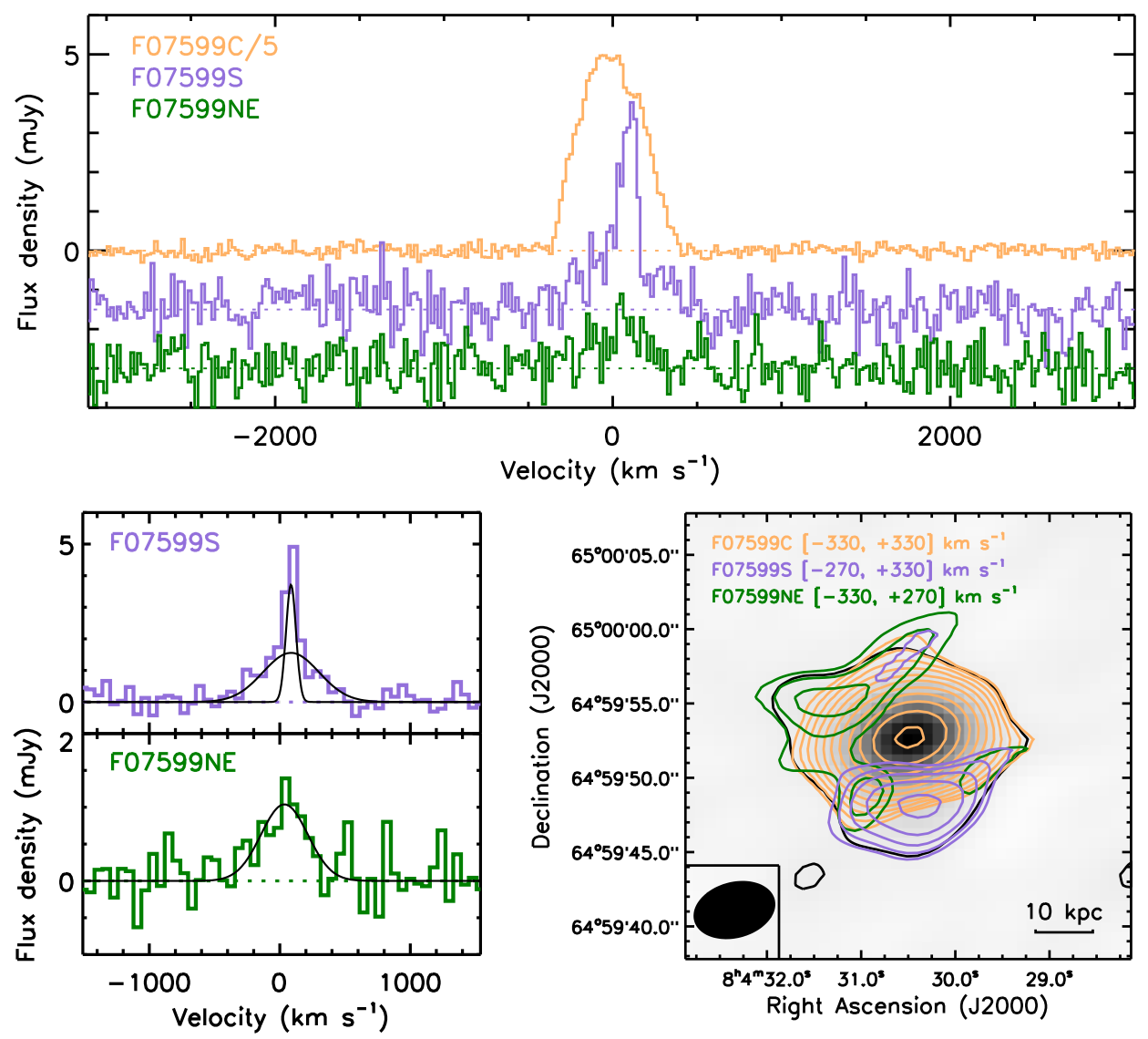

Figure 4. Top: $\mathrm{CO}(1-0)$ spectra of the three components extracted from GILDAS task uv_fit using an elliptical Gaussian and two point-source functions for F07599C (orange), F07599S (purple) and F07599NE (green), respectively. The CO spectrum of F07599C is divided by 5 for comparison purpose. All spectra are smoothed to a velocity resolution of $\sim 20 \mathrm{~km} \mathrm{~s}{ }^{-1}$. Bottom-left: zoomed-in views of $\mathrm{CO}(1-0)$ spectra for F07599S and F07599NE and the spectra are re-binned in channels of $\sim 60 \mathrm{~km} \mathrm{~s}{ }^{-1}$. The black lines show the Gaussian fits to the spectra. For F07599S, we modeled the line profile with two Gaussians (one narrow and one broad), while for F07599NE the spectrum is fitted by using a single Gaussian. Bottom-right: the corresponding continuum-subtracted $\mathrm{CO}(1-0)$ maps (colored contours) of the three components overlaid on the $\mathrm{CO}(1-0)$ map of the whole IRAS F07599+6508 (greyscale; same as the map in the right panel of Figure 2). The colored contours show the CO(1-0) emission integrated in the $[-330,+330] \mathrm{km} \mathrm{s}^{-1}$ (orange), $[-270,+330] \mathrm{km} \mathrm{s}^{-1}$ (purple), and $[-330,+270] \mathrm{km} \mathrm{s}^{-1}$ (green) velocity range, respectively, while the black contour indicates the global $\mathrm{CO}(1-0)$ emission detected at a $3 \sigma$ significance in the intensity map integrated in the $[-630,+450] \mathrm{km} \mathrm{s}^{-1}$ velocity range. The colored contours start at $2 \sigma$ and increase by a factor of 1.5 . The $1 \sigma \mathrm{rms}$ noise is $0.075,0.095$, and $0.082 \mathrm{Jy} \mathrm{beam}^{-1} \mathrm{~km} \mathrm{~s}^{-1}$ for F07599C, F07599S, and F07599NE, respectively. The CO map of F07599C was derived from a cleaned data cube, while the maps of F07599S and F07599NE were not cleaned given the relatively low SNR.

F07599NE) are identified at $16.0 \sigma$ and $6.4 \sigma$ in the intensity maps of residual data cube, respectively. These weak features are also clearly present in the channel maps, where extended structures are found in the $\mathrm{S}$ and NE direction (see Appendix B).

Figure 5 shows the multi-wavelength images of IRAS F07599+6508 in SDSS $u_{-}, g_{-}, r_{-}, i_{-}, z_{-}$, and PanSTARRS1 $y$-bands, and an overlay of the CO $(1-0)$ emission of the three decomposed components on the HST/WFPC2 F702W optical image. The optical morphologies shown in gri and HST images are found to be complex with tidal-like features, indicative of an asymmetric stellar distribution, while the ultraviolet (UV; $u$-band at $\sim 0.35 \mu \mathrm{m})$ and near-IR $(z$ - and $y$-bands at $\sim 1 \mu \mathrm{m}$ ) emission show more regular and compact distribution. A consistent morphology structure in the UV band has been presented by Surace \& Sanders (2000). The tidal features seen in the optical is probably connected with an ongoing or past merging event in IRAS F07599+6508. Moreover, the absence of tidal tails observed in the near-IR bands is likely to suggest that the emission from optical tidal tails may trace the massive, young stellar population formed at recent epochs, since the near-IR emission is mostly sensitive to the longerlived, low-mass stars. If this is indeed the case, it will also explain the morphology observed in UV band where 

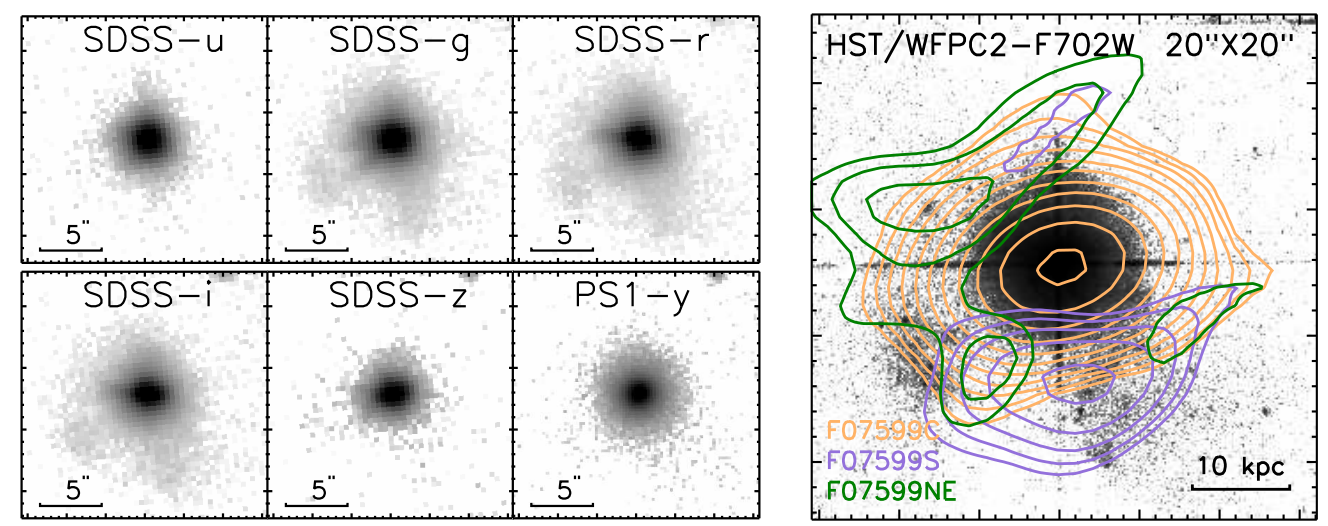

Figure 5. Left: optical images of IRAS F07599+6508. From left to right and top to bottom, we show the SDSS $u$-, $g$-, $r$-, $i$-, $z$-band, and Pan-STARRS1 $y$-band images. Right: overlay of $\mathrm{CO}(1-0)$ contours (colored) of the three components on the HST/WFPC2 F702W band image. The $\mathrm{CO}(1-0)$ contours are at the same levels as in Figure 4 . The optical diffraction spikes in the middle of the object reflect the central luminous point source of AGN that is significantly brighter than its host galaxy. Both the SDSS gri and HST optical images show clear signs of extended structure to the southwest and southeast with respect to the main source.

the emission is largely attenuated in dust obscured regions.

Interestingly, by comparing the distribution of $\mathrm{CO}$ molecular gas and stellar emission (see the right panel of Figure 5), we find that the tidal feature in the optical is coincident with the CO substructure F07599S. This provides evidence that the $\mathrm{CO}$ feature observed in the $\mathrm{S}$ region may be a real structure. Moreover, the $\mathrm{CO}$ emission also show an extension to the southeast (SE), the same direction as the other tidal-tail-like feature observed in the optical images, although the CO extended structure toward the SE appear in only a few adjacent channels (e.g., channel maps at the velocity of about $-178 \mathrm{~km} \mathrm{~s}^{-1}$ and $-58 \mathrm{~km} \mathrm{~s}^{-1}$; see Appendix B). Meanwhile, a faint peak coincident with the location of the $\mathrm{CO}$ peak in F07599NE is identified in the HST image, indicative of a possible association.

\subsubsection{CO kinematics}

In Figure 6 we show the velocity and velocity dispersion maps of $\mathrm{CO}(1-0)$ emission within $[-330,+330] \mathrm{km} \mathrm{s}^{-1}$ velocity range for the whole galaxy IRAS F07599+6508 and the three decomposed components, respectively. These maps were created by applying a flux threshold of $3 \sigma$. The velocity map of the whole galaxy IRAS F07599+6508 shows irregular structure, indicative of complex velocity distribution. For the main component F07599C that is decomposed from the whole system, a systematic velocity gradient seems to be present in the north-south direction, from about -70 to $+70 \mathrm{~km} \mathrm{~s}^{-1}$, although the gas at the edge to the southeast and west appear to be disturbed. This implies that the bulk of molecular gas is likely rotationally supported. However, the spatial resolution of our data is not adequate to unambiguously interpret the observed velocity gradient as due to disk rotation. An alternative possibility could be that a velocity gradient and broad line profile may originate from an ongoing merger, as the velocity gradient is observed roughly along the direction connecting F07599C and F07599S. For F07599S, a velocity gradient of $\sim 180 \mathrm{~km} \mathrm{~s}^{-1}$ (from about -70 to $+110 \mathrm{~km} \mathrm{~s}^{-1}$ ) is also observed in the north-south direction, while no well-defined structure is visible in the velocity map of F07599NE, in which the velocity is positive around $60-90 \mathrm{~km} \mathrm{~s}^{-1}$ with respect to the QSO redshift.

If we assume a rotating disk geometry for the main component F07599C, we can estimate the dynamical mass within the CO-emitting region as $M_{\mathrm{dyn}} / M_{\odot} \approx$ $2.3 \times 10^{5} v_{\text {cir }}^{2} R$ (e.g., Wang et al. 2010), where $R$ is the $\mathrm{CO}$ disk radius of $4.6 \mathrm{kpc}$ (0.75 times the CO FWHM major axis from visibility fitting; see Table 1 ) and $v_{\text {cir }}$ is the maximum circular velocity of the gas disk in $\mathrm{km} \mathrm{s}^{-1}$. The $v_{\text {cir }}$ is estimated as $v_{\text {cir }}=0.75 \Delta v_{\mathrm{FWHM}} / \sin i$ (Ho 2007), where $i$ is the inclination angle between the polar axis of disk and the line of sight and can be roughly calculated from the $\mathrm{CO}$ minor and major axis ratio $\left(a_{\mathrm{min}} / a_{\mathrm{maj}}\right.$; see Table 1$)$, i.e., $i=\cos ^{-1}\left(a_{\mathrm{min}} / a_{\mathrm{maj}}\right)=$ $45^{\circ}$. The derived $M_{\text {dyn }}$ is $2.1 \times 10^{11} M_{\odot}$ with a $v_{\text {cir }}$ of $450 \mathrm{~km} \mathrm{~s}^{-1}$ for the primary disk F07599C. We stress that this estimate of dynamical mass can be significantly affected by the large uncertainties of source size, inclination angle, and the assumption of an inclined disk geometry. The total amount of molecular gas in the host galaxy is $M_{\text {tot,mol }} \sim 1.1 \times 10^{10} M_{\odot}$ by adopting a classic ULIRG-like $\mathrm{CO}$-to- $\mathrm{H}_{2}$ conversion factor of $\alpha_{\mathrm{CO}}=0.8 M_{\odot}\left(\mathrm{K} \mathrm{km} \mathrm{s}^{-1} \mathrm{pc}^{-2}\right)^{-1}$. We obtain a molecular gas fraction of $\sim 0.05$ for IRAS F07599+6508 by assuming the gas fraction can be approximated as the ratio of gas to dynamical mass, slightly lower than those (median value of $0.22 \pm 0.04$ ) of IR QSOs measured by Tan et al. (2019), and the local ULIRGs as well (Downes \& Solomon 1998). Here we note the large 


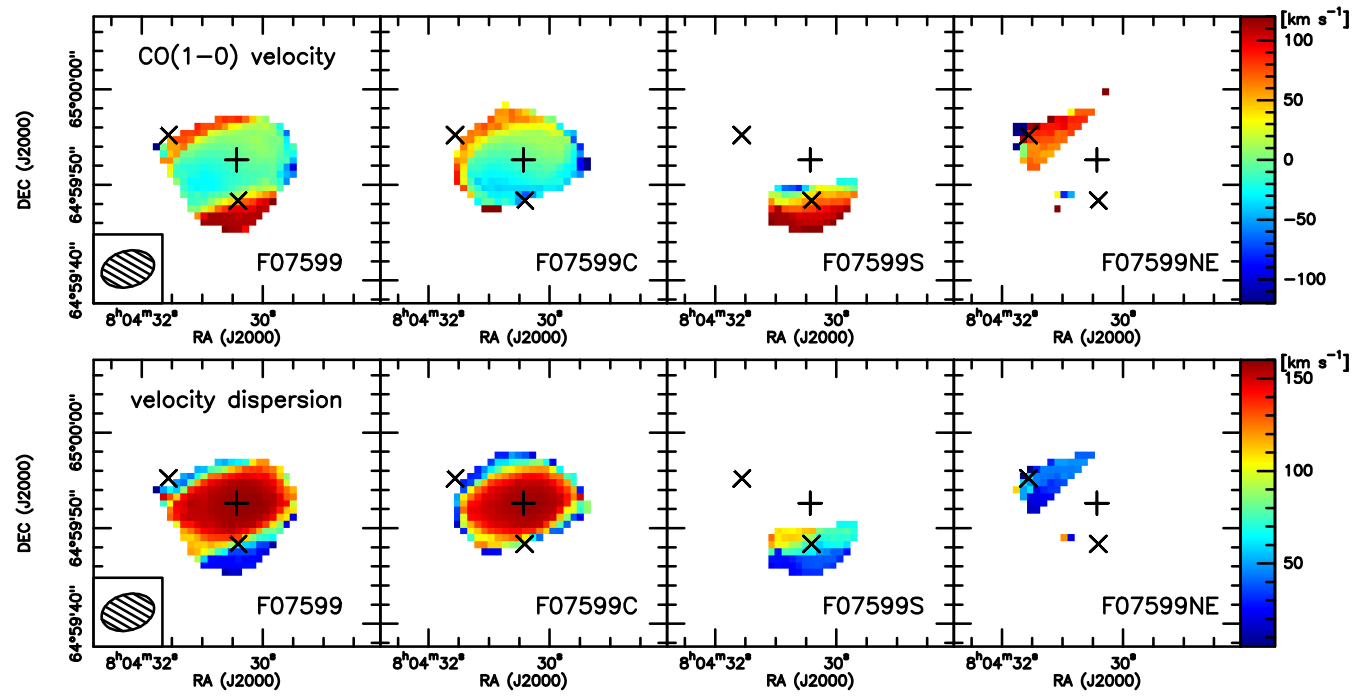

Figure 6. $\mathrm{CO}(1-0)$ velocity field (top) and velocity dispersion (bottom) maps for the whole galaxy IRAS F07599+6508 (first column) and the three subcomponents, F07599C (second column), F07599S (third column), and F07599NE (fourth column). The CO velocity and velocity dispersion maps are calculated using pixels deteccted at $\geqslant 3 \sigma$ and are masked where the integrated intensity is $<3 \sigma$ in each component. The plus sign marks the peak of $3 \mathrm{~mm}$ continuum emission, while the two crosses indicate the CO peak of F07599S and F07599NE, respectively. The synthesized beam is shown in the bottom-left corner of the first column panel.

uncertainties involved in the estimate of molecular gas mass, due to the assumption of conversion factor $\alpha_{\mathrm{CO}}$, which is uncertain and varies significantly from source to source (Bolatto et al. 2013). Recent studies show evidence for higher $\alpha_{\mathrm{CO}}$ values in (U)LIRGs that are affected by outflows if a substantial amount of molecular gas is in dense, gravitationally bound states (e.g., Cicone et al. 2018, and references therein).

The velocity dispersion map shows $\sigma_{\mathrm{v}} \sim 160 \mathrm{~km} \mathrm{~s}^{-1}$ at the center of IRAS F07599+6508, which is significantly higher than the typical peak dispersion of $\sim 80 \mathrm{~km} \mathrm{~s}^{-1}$ observed in local IR QSOs (Tan et al. 2019), and $z \gtrsim 6$ QSOs $\left(\sim 130 \mathrm{~km} \mathrm{~s}^{-1}\right)$ as well (Neeleman et al. 2021). A plausible explanation could be related to the beam smearing effect that increases the observed dispersion, since the spatial angular resolution of our NOEMA observations is about an order of magnitude lower than the ALMA observations $\left(\sim 0 .^{\prime \prime} 5\right)$ shown by Tan et al. (2019). Assuming a uniform intrinsic dispersion across the galaxy disk of IRAS F07599+6508, we then estimate a dispersion of $\sigma_{\mathrm{v}} \sim 70-100 \mathrm{~km} \mathrm{~s}^{-1}$, which is measured in the outer region of $\mathrm{F} 07599 \mathrm{C}$ where the beam smearing is considered to be less severe (Davies et al. 2011). The velocity dispersion map of F07599S shows an increase in the north direction with a peak dispersion of $\sim 110 \mathrm{~km} \mathrm{~s}^{-1}$ which is in the overlap region between F07599C and F07599S, while the gas in F07599NE appears less perturbed with dispersion of $\sim 30-60 \mathrm{~km} \mathrm{~s}^{-1}$. The increased velocity dispersion observed in the overlap region could be interpreted as a turbulence-dominated shock region induced by a merger or a possible $\mathrm{CO}$ outflow component. Higher resolution observations are needed to better constrain the intrinsic velocity dispersion.

\subsection{Radio Continuum Emission}

In star-forming galaxies, the radio emission is dominated by synchrotron radiation from relativistic electrons accelerated in supernovae remnants of massive stars. It is largely optically thin and unaffected by dust extinction, and therefore an excellent probe of very recent star formation activity in galaxies (Condon 1992). For the radio-quite (RQ) AGNs, which represent the majority of the AGN population, the origin of radio emission could be connected with a wide range of possible mechanisms, namely, star formation, AGN-driven wind, jets, and accretion-disk coronae (Panessa et al. 2019).

Figure 7 shows the VLA $1.49 \mathrm{GHz}$ contour maps overlaid on the NOEMA $\mathrm{CO}(1-0)$ data that is decomposed into three components. Along the direction of the minor axis of synthesized beam of $1.49 \mathrm{GHz}$ observations, two clumpy structures outside the central bright compact radio source are potentially detected at $\sim 3 \sigma$ and $\sim 4 \sigma$ level to the $\mathrm{S}$ and NE direction, respectively. The radio peaks of both the central compact source and the two clumps are well aligned with the $\mathrm{CO}$ peaks of the three decomposed components, reinforcing the fact that the faint $\mathrm{CO}$ features observed in the $\mathrm{S}$ and NE direction are very likely to be real.

A 2D Gaussian fit to the $1.49 \mathrm{GHz}$ image returns a peak flux density of $32.33 \pm 0.10 \mathrm{mJy}^{\text {beam }}{ }^{-1}$ and a consistent integrated flux density of $32.43 \pm 0.19 \mathrm{mJy}$, indicative of a relatively compact structure of radio emission. This measurement agrees well with the $1.4 \mathrm{GHz}$ flux density of $39.5 \mathrm{mJy}$ derived from the NRAO VLA 


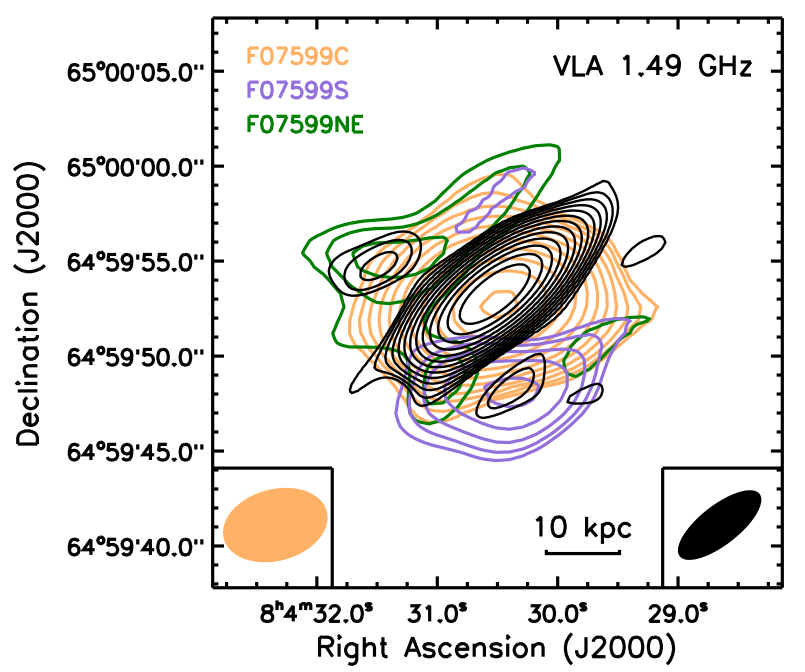

Figure 7. VLA $1.49 \mathrm{GHz}$ contour (black) maps of IRAS F07599+6508 overlaid on the $\mathrm{CO}(1-0)$ contour (colored) maps of the three components. The $\mathrm{CO}(1-0)$ contours are at the same levels as in Figure 4. The contours of 1.49 $\mathrm{GHz}$ continuum emission start at $2 \sigma$ ( $1 \sigma$ noise level is 74.9 $\mu \mathrm{Jy}$ beam $^{-1}$ ) and increase by a factor of 1.5 . The solid orange and black ellipses in the bottom left and right corners are the beam size of $\mathrm{CO}$ and $1.49 \mathrm{GHz}$ maps, with angular

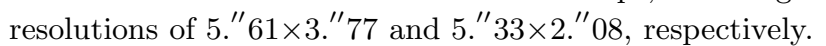

Sky Survey (Condon et al. 1998). IRAS F07599+6508 is additionally detected at 4.86, 8.44, 14.94, and 22.46 $\mathrm{GHz}$ by VLA. The maps and the flux measurements at these radio frequencies are shown in Appendix C. At the redshift $z=0.1486$, observations at the radio frequencies listed in Table 2 sample $\sim 1.7,5.6,9.7,17.2$, and $25.8 \mathrm{GHz}$ in the rest-frame, respectively. In the following section, we adopt the rest-frame frequencies for clarity.

Figure 8 shows the SED of the continuum emission at radio and $\mathrm{mm}$ bands in IRAS F07599+6508. At $\mathrm{GHz}$ frequencies, the flux density $S_{\nu}$ in terms of the observed frequency $\nu$ is generally defined as $S_{\nu} \propto \nu^{\alpha}$, with $\alpha$ the radio spectral index. The spectral indices measured from 1.7-5.6, 5.6-9.7, 9.7-17.2, and 17.2-25.8 $\mathrm{GHz}$ are $\alpha_{5.6}^{1.7}=-1.10 \pm 0.09, \alpha_{9.7}^{5.6}=-1.16 \pm 0.22$, $\alpha_{17.2}^{9.7}=-1.18 \pm 0.26$, and $\alpha_{25.8}^{17.2}=-0.34 \pm 0.78$, respectively. It is clear that the spectrum turns over from steep to flat at $17.2-25.8 \mathrm{GHz}$, which is consistent with the expectation that the contribution of thermal emission increases at higher frequencies. At low frequencies, the spectral index $\alpha_{5.6}^{1.7}$ is slightly flatter than those at higher frequencies, $\alpha_{9.7}^{5.6}$ and $\alpha_{17.2}^{9.7}$, both of which show almost identical values. The flatter index observed at lower frequencies has been interpreted as a result of optical depth effects that the radio spectrum typically shows a sharp decline due to free-free absorption (e.g., Condon 1992; Clemens et al. 2010). In contrast, the

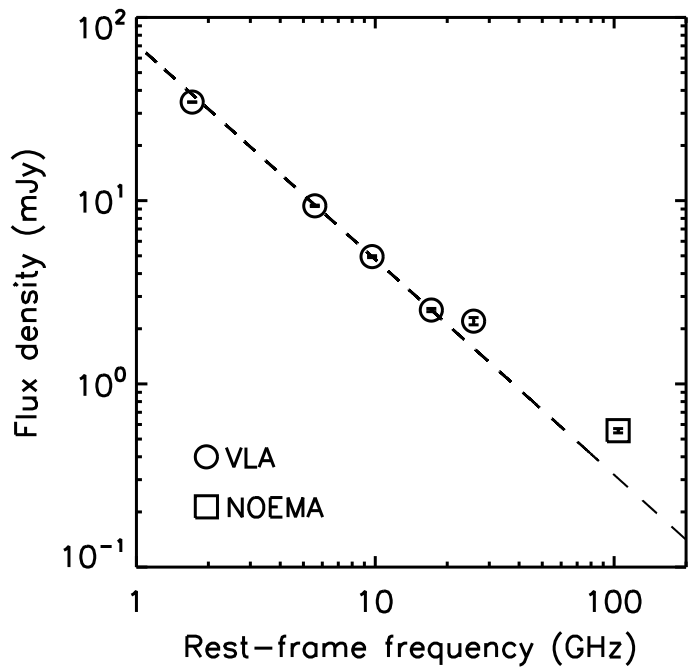

Figure 8. The radio-to-mm spectral energy distribution of IRAS F07599+6508 based on the data from VLA (circle) archive and NOEMA (square).

flux at larger frequency should be much less affected (e.g., Clemens et al. 2008). Therefore, we assume a synchrotron (non-thermal) spectral index of $\alpha^{\mathrm{NT}}=-1.18$, equivalent to the steepest part of the radio spectrum measured between 9.7 and $17.2 \mathrm{GHz}$ (see the dashed line in Figure 8). This slope is slightly steeper than a normal synchrotron spectral index of $\alpha=-0.8$ derived for a normal star-forming galaxy (Condon 1992), but consistent with the slopes measured for RQ AGN (e.g., Behar et al. 2015).

We extrapolate the contribution of non-thermal emission from $\alpha_{17.2}^{9.7}$ at the NOEMA frequency of $90.5 \mathrm{GHz}$ ( $\sim 103.9 \mathrm{GHz}$ in the rest-frame) and subtract from the observed flux density. In addition, subtracting off an estimate of the thermal dust contribution to the 103.9 $\mathrm{GHz}$, which is derived from a grey-body model fit to the Hershel/SPIRE data with an emissivity spectral index $\beta=1.6$ (Clements et al. 2018), we obtain an estimate of free-free emission component of $0.17 \mathrm{mJy}$. This implies a free-free radio fraction at $103.9 \mathrm{GHz}$ of $\sim 30 \%$. A similar procedure applied to $25.8 \mathrm{GHz}$ data leads to a freefree fraction of $\sim 30 \%$ estimated at this frequency. This is consistent with the median thermal fraction of 20$30 \%$ at $22 \mathrm{GHz}$ measured for local ULIRGs (Vega et al. 2008).

We derive an extrapolated $1.4 \mathrm{GHz}$ flux density of 43.4 mJy from the observed rest-frame $1.7 \mathrm{GHz}$ flux density by assuming a radio power-law index of -1.18. Using the flux densities measured at IRAS 60 and $100 \mu \mathrm{m}$, we 
calculate a FIR-radio flux $\operatorname{ratio}^{4} q=1.67$. This implies a linear ratio about 4 times smaller than the average value of $q=2.34 \pm 0.01$ found for local star-forming galaxies (Yun et al. 2001), possibly suggestive of an additional contribution from compact AGN source that boosting the radio flux.

\section{DISCUSSION}

The main results of our NOEMA observations are the detection of $3 \mathrm{~mm}$ continuum emission and the multiple spatially decomposed $\mathrm{CO}$ components in the host galaxy of IRAS F07599+6508. By combining with multi-wavelength data, we begin the discussion by looking into the properties and the possible origin of these components.

\subsection{Nature of the 3mm Emission}

The $3 \mathrm{~mm}$ continuum emission of IRAS F07599+6508 is resolved with a deconvolved size of $1 .^{\prime \prime} 5(\sim 4.0 \mathrm{kpc})$. Assuming a non-thermal spectral index of $\alpha^{\mathrm{NT}}=-1.18$, we find that about $30 \%$ of the emission at $3 \mathrm{~mm}$ arises from a thermal free-free component. This is significantly lower than the thermal fraction of $\sim 70 \%$ at $3 \mathrm{~mm}$ expected for normal star-forming galaxies (Condon 1992), indicating that the nonthermal synchrotron component from AGN may contribute substantially to the $3 \mathrm{~mm}$ emission in IRAS F07599+6508. This could be expected since it does not follow the FIR-radio correlation characteristic of star-forming galaxies, with a ratio of $L_{\mathrm{FIR}} / L_{1.4 \mathrm{GHz}}$ being about four times lower. A similar situation has been observed in Mrk 231, the nearest IR QSO with a compact radio core(e.g., Lonsdale et al. 2003), where a rather low thermal fraction is observed at $33 \mathrm{GHz}$ (Barcos-Muñoz et al. 2017). As mentioned above, an additional nonthermal component from AGN could contribute to the emission at high radio frequencies. An alternative explanation for the observed low thermal fraction is connected with the possibility that, the production of free-free emission might be suppressed due to the absorption of ionizing stellar photons by dust that concentrates in the dense starburst regions.

Taking the 2-10 keV X-ray luminosity from Brightman \& Nandra (2011), we find a radio $(\sim 3 \mathrm{~mm})$ luminosity to X-ray luminosity ratio of $L_{\mathrm{mm}} / L_{\mathrm{X}} \sim$ $3 \times 10^{-2}$ for IRAS F07599+6508. This value is about two orders of magnitude lower than those of radio-loud (RL) AGNs, which typically have powerful relativistic jets that shine in the radio band, but close to the ratios $\left(10^{-2}-10^{-4}\right)$ measured for RQ AGNs (Behar et al. 2015; Panessa et al. 2019), suggestive of a different origin of radio emission compared with RL QSOs. We

\footnotetext{
${ }^{4}$ The FIR-radio flux ratio $q$ is defined as the ratio of the FIR luminosity $(40-120 \mu \mathrm{m})$ to the radio power (Condon 1992):$$
q \equiv \log _{10}\left(\frac{L_{\mathrm{FIR}}}{3.75 \times 10^{12} \mathrm{~W}}\right)-\log _{10}\left(\frac{L_{1.4 \mathrm{GHz}}}{\mathrm{W} \mathrm{Hz}^{-1}}\right)
$$

note that the ratio of $L_{\mathrm{mm}} / L_{\mathrm{X}} \sim 3 \times 10^{-2}$ derived here could be an overestimated value, given that the AGN embedded in the galaxy IRAS F07599+6508 is known to be heavily obscured and thus a portion of X-ray photons may be absorbed (see Luo et al. 2014; La Caria et al. 2019). A connection between the radio emission and the nuclear accretion disk has been proposed to explain the correlation of the radio luminosity $L_{\mathrm{mm}}$ and the X-ray luminosity $L_{\mathrm{X}}$ observed in RQ AGNs (e.g., Laor \& Behar 2008). More evidences for an X-ray corona (i.e., hot plasma with a temperature of $\sim 10^{9} \mathrm{~K}$ ) origin of radio emission in RQ AGNs have been presented by the detections of millimeter excess (e.g., Behar et al. 2015; Inoue \& Doi 2018). These are in line with the theoretical prediction for a radio synchrotron component from X-ray corona in the vicinity of black hole accretion disks contributing to the emission in the millimeter band (see Inoue \& Doi 2014; Raginski \& Laor 2016). Nevertheless, a conclusive evidence for the coronal radio emission would be the detection of the radio/millimeter and X-ray variability correlation similar to that observed in stellar coronae (Panessa et al. 2019).

The thermal free-free emission arises directly from HII regions ionized by massive stars and its intensity is proportional to the ionizing photon rate from young starforming regions, and thereby provide an excellent diagnostic for the current SFR of galaxies (Condon 1992). Taking the free-free emission flux measured at rest-frame $103.9 \mathrm{GHz}$ and the free-free SFR calibration given in Murphy et al. (2012), we calculate an SFR of $77 M_{\odot}$ $\mathrm{yr}^{-1}$ for IRAS F07599+6508 by assuming a thermal spectral index of -0.1 and an electron temperature of $T_{\mathrm{e}}=10^{4} \mathrm{~K}$. Compared to the IR-derived SFR of $280 \mathrm{M}_{\odot}$ $\mathrm{yr}^{-1}$ that is estimated based on the heated dust component (Hao et al. 2008), a lower SFR measured by the free-free emission could be expected since free-free emission is sensitive to massive stars with ages $\lesssim 10 \mathrm{Myr}$ and consequently probe the very recent star formation activity. In addition, the free-free-derived SFR would be underestimated if a significant fraction of ionizing photons are absorbed by dust. Alternatively, it could be that a portion of IR luminosity arise from the dust heating by nuclear luminous AGN, which would lead to an overestimate of SFR. Indeed, we obtain an SFR of $150 M_{\odot} \mathrm{yr}^{-1}$ using the calibration given by Murphy et al. (2012) by correcting for the AGN contribution to the bolometric luminosity $\left(\alpha_{\mathrm{AGN}}=0.75\right.$; Veilleux et al. 2009), which is close to the free-free SFR estimate. All of these including the uncertainties (e.g., variations in the actual $T_{\mathrm{e}}$ ) on the estimation of free-free-derived SFR may explain the discrepancy in SFR estimates.

\subsection{Origin of the off-center $C O$ components}

By exploiting the CO line cube of IRAS F07599+6508, we have identified two off-center CO subcomponents, 
F07599S and F07599NE, at a significance level of $16.0 \sigma$ and $6.4 \sigma$ in the intensity map, respectively. Figure 4 shows the $\mathrm{CO}(1-0)$ spectra and maps extracted for these two subcomponents and the main component F07599C as well. F07599S and F07599NE are located to the south and northeast of the disk component with a projected distance of $\sim 11.4$ and $19.1 \mathrm{kpc}$ and with a fraction of $9 \%$ and $4 \%$ of CO flux contributed to the total CO flux, respectively. These two CO subcomponents are not detected in $3 \mathrm{~mm}$ continuum emission. For the main component F07599C, the systematic CO velocity gradient observed may be a signature of a rotating disk structure. If confirmed, the source size of $\sim 6.1 \mathrm{kpc}$ measured would suggest that the $\mathrm{CO}$ disk is very extended, which is in sharp contrast with local ULIRGs where the majority of molecular gas is centrally concentrated within a radius of $\sim 1 \mathrm{kpc}$ (Downes \& Solomon 1998; Wilson et al. 2008).

The optical images of IRAS F07599+6508 show evidence for a tidal-tail-like structure (see Figure 5), suggestive of a recent merging event in IRAS F07599+6508. It is interesting to note that the tidal feature shown in the optical image is spatially coincident with the $\mathrm{CO}$ feature detected in the $\mathrm{S}$ region. In addition, a faint peak coincident with the location of the $\mathrm{CO}$ peak in F07599NE is identified in the HST optical image, likely associated with the origin of the CO plume feature observed in the $\mathrm{NE}$ direction. It can be seen from the channel maps in Appendix B that the CO emission is extended to the $\mathrm{S}$ and $\mathrm{NE}$ direction, suggesting that the $\mathrm{CO}$ emission identified in the extranuclear region of F07599S and F07599NE are very likely to be real components. Furthermore, the two potentially detected clumpy structures revealed in the $1.49 \mathrm{GHz}$ radio image are found to align well with the peaks of F07599S and F07599NE (see Figure 7), as well as the faint substructures detected at 4.86 and $8.44 \mathrm{GHz}$ (see Appendix C), supporting for the reality of such CO feature.

In Figure 4 we have shown that the $\mathrm{CO}(1-0)$ spectrum extracted for F07599S is best-fitted with a twocomponent (narrow and broad) Gaussian. The detection of narrow (FWHM $=100 \pm 10 \mathrm{~km} \mathrm{~s}^{-1}$ ) feature may indicate the presence of extended dynamical "quiescent" $\mathrm{CO}$ gas. For the broad component, the Gaussian fit gives an FWHM of $500 \pm 80 \mathrm{~km} \mathrm{~s}^{-1}$, slightly larger than the line FWHM $\left(430 \pm 10 \mathrm{~km} \mathrm{~s}^{-1}\right)$ of the primary component F07599C. Together with the shift in velocity $(\sim 100$ $\mathrm{km} \mathrm{s}^{-1}$ ) and the tidal-tail-like structure observed in the optical image, it is likely that F07599S represents a separate component from main galaxy, and has a merger origin. One of the possibilities is that F07599S may trace the emission from a small obscured companion to the host galaxy of IRAS F07599+6508. The non-detection of UV emission in F07599S show evidence for the large attenuation by dust (see Figure 5). More evidence for a merger origin is given by the increased velocity dis- persion observed in the overlap region between F07599S and the main component, which could be interpreted as a turbulence-dominated shock region induced by an interaction.

For F07599NE, the best-fit of the $\mathrm{CO}(1-0)$ line is a single Gaussian with both FWHM and peak velocity consistent within the errors with the main component. The significant lower velocity dispersion observed in this component imply that F07599NE may have a different origin from F07599S. Similar gas substructure has been observed in high- $z$ starbursts in [C II] emission but with a much smaller physical separation $(\sim 2$ kpc; Tadaki et al. 2020), where the formation of subcomponent is expected to be associated with a gravitationally unstable disk or shocks. By contrast, the large physical separation (projected distance of $\sim 19 \mathrm{kpc}$ ) observed between F07599NE and the galaxy nucleus indicates that the $\mathrm{CO}$ plume feature observed in the NE direction could be a separate component, likely associated with the tidal debris reminiscent of a previous merger. Similar results have been seen in the IR QSO IRAS F23411+0228, where an off-center clumpy structure is detected in both $\mathrm{CO}$ line and $3 \mathrm{~mm}$ continuum maps and is found to align with a faint peak identified in the HST image, which was interpreted as either an ongoing merger or a clumpy star-forming region at large radii (Tan et al. 2019). Cicone et al. (2015) have shown a very extended (up to $r \sim 30 \mathrm{kpc}$ ) [C II] cold gas component observed in a $z>6$ QSO host galaxy and they interpret that the extended [C II] emission may arise from a different ISM phase than dense photon-dominated regions.

We note that there is a source accompanying IRAS F07599+6508 in multi-wavelength data set (from GALEX near-UV to WISE $3.4 \mu \mathrm{m}$ bands; see Appendix D), with a distance of $17 .{ }^{\prime \prime} 5$ (projected distance of $\sim 45 \mathrm{kpc}$ ) to the northwest. This source has a cross-identification as WISEA J080428.28+650001.7 (hereafter J0804+6500) in the NASA/IPAC ${ }^{5}$ Extragalactic Database. There is no optical spectroscopic data available for this object. In Appendix D, we show the NOEMA spectrum extracted from the position of J0804+6500 by fitting the visibilites with a point source. We do not detect any significant emission in either continuum or line from this source. With the data available, it is not possible to assess whether J0804+6500 is physically related with the IRAS F07599+6508 system, or is only a projected companion source. The detection of line emission from this source would help us measure the redshift and further determine whether it is an interacting companion of IRAS F07599+6508.

Together with the CO clumpy structures of local IR QSOs revealed with ALMA high resolution observations (Tan et al. 2019), the multiple components revealed in

\footnotetext{
${ }^{5}$ http://ned.ipac.caltech.edu
} 
the CO maps show evidence for the complex morphology that may associate with the intense star formation and AGN activities. However, additional deep CO observations with higher angular resolution are needed for confirming the faint features observed in F07599S and F07599NE and testing the corresponding hypothesis.

\subsection{Comparison of molecular gas with Mrk 231}

As noted previously in Section 1, IRAS F07599+6508 and Mrk 231 share many common or similar properties in multi-wavelength, i.e., X-ray, UV, optical, and IR, and have been proposed as local templates of candidate ULIRG-to-optical QSO transition objects. Highresolution $\mathrm{CO}$ observations of Mrk 231 show that the molecular gas is concentrated in the central $\sim 1 \mathrm{kpc}$ rotating disk (Downes \& Solomon 1998; Cicone et al. 2012; Feruglio et al. 2015), though $\mathrm{O}_{2}$ emission might be present over $\sim 10 \mathrm{kpc}$ (Wang et al. 2020). Compared with Mrk 231, the molecular gas of IRAS F07599+6508 is also likely distributed in a disk with systematic velocity gradient in the main component, but much more extended with a size of $\sim 6.1 \mathrm{kpc}$. In addition, the CO line width of IRAS F07599+6508 is $\sim 430 \mathrm{~km} \mathrm{~s}^{-1}$ FWHM, much broader than that of $190 \mathrm{~km} \mathrm{~s}^{-1}$ measured for the core component of Mrk 231 (Downes \& Solomon 1998). The $\mathrm{CO}(2-1)$ to $\mathrm{CO}(1-0)$ line luminosity ratios derived for IRAS F07599+6508 and Mrk 231 are both $\sim 0.8$ (Xia et al. 2012; Cicone et al. 2012), indicative of modest sub-thermal molecular gas in their hosts.

The detection of massive, highly energetic, and kpcscale molecular outflows in Mrk 231 show direct observational evidence for AGN feedback onto the host galaxy (e.g., Feruglio et al. 2010, 2015; Aalto et al. 2012; Cicone et al. 2012), consistent with the AGNgalaxy co-evolutionary models which propose that the outflow feedback is an important process in quenching the star formation activity in massive galaxies (e.g., Di Matteo et al. 2005; Hopkins et al. 2006). The $\mathrm{CO}(1-0)$ wing-to-peak ratio in Mrk 231 is $\sim 2.5 \%$, in agreement with the ratio of 1-8\% typically measured for local ULIRGs (Feruglio et al. 2010; Cicone et al. 2012, 2014).

The presence of molecular outflow in IRAS F07599+6508 has been confirmed by the detection of $\mathrm{OH} 119 \mu \mathrm{m}$ P-Cygni profile with velocities $<1000$ $\mathrm{km} \mathrm{s}^{-1}$ (Veilleux et al. 2013). A fast wind has also been seen in this system in the ionized gas as traced by the broad blueshift optical spectra $(\mathrm{H} \alpha$ and $\mathrm{H} \beta)$ and in the neutral atomic phase with broad absorption observed in Na I D line (e.g., Zheng et al. 2002; Rupke et al. 2017).

In the left-top panel of Figure 9 we show the continuum-subtracted NOEMA $\mathrm{CO}(1-0)$ spectrum of the whole galaxy of IRAS F07599+6508, which is obtained by summing up the flux of the three decomposed components in each channel, compared with the $\mathrm{CO}(1-$ 0) line observed with the IRAM 30m (Xia et al. 2012). The CO peak flux measured by NOEMA is found to agree well with the IRAM 30m data, suggesting that little emission is filtered out by our interferometric observations. The residual spectrum after removing a Gaussian fit to the core component of the $\mathrm{CO}$ spectrum is shown in the left-bottom panel of Figure 9. A noise level $(1 \sigma)$ of $0.68 \mathrm{mJy}$ in each $\sim 60 \mathrm{~km} \mathrm{~s}^{-1}$ channel was measured, corresponding to $2.3 \%$ of the peak flux density. Scaling to the signal strengths from Mrk 231 would lead to an estimated flux density of the CO broad emission of $\sim 0.75$ mJy for IRAS F07599+6508, comparable to the noise level of our NOEMA data.

It is interesting to note that several consecutive channels in the blue-shifted side of the CO spectrum (see Figure 9, left-bottom) show flux density higher than $1 \sigma$. We averaged the visibilities of the high-velocity component in the velocity range $[-1340,-2000] \mathrm{km} \mathrm{s}^{-1}$ and fitted a point-source model. The peak of highvelocity $\mathrm{CO}$ emission is located between the F07599C and F07599S with a projected distance of $4 .{ }^{\prime \prime} 3$ from the galaxy nucleus (see Figure 9, right). The flux derived from uv_fit is $0.228 \pm 0.054 \mathrm{Jy} \mathrm{km} \mathrm{s}^{-1}$, corresponding to a detection significance of $4.2 \sigma$. However, this does not satisfy the criterion defined by Cicone et al. (2014), i.e., $\mathrm{CO}$ emission with velocities $>500 \mathrm{~km} \mathrm{~s}^{-1}$ is detected with a significance of at least $5 \sigma$, for claiming the detection of a molecular outflow. The $\mathrm{OH}$ outflow counterpart in $\mathrm{CO}$ emission is not significantly detected in our NOEMA data, although there is a hint of some flux at very high blueshifted velocities in the overlap region between F07599C and F07599S (see Figure 9), where the extracted CO spectrum shows a clear decomposition into broad plus narrow components (see the CO spectrum of F07599S in Figure 4). Nevertheless, it is difficult to rule out the existence of a weak $\mathrm{CO}$ outflow with the current data.

Given the much extended $\mathrm{CO}$ disk providing fuel for active large-scale star formation in IRAS F07599+6508, perhaps a much weaker outflow feedback in quenching star formation is expected here as compared to that of Mrk 231, where bursts of star formation only remain in the central compact core. If that is true, IRAS F07599+6508 should be in an earlier phase than that of Mrk 231 in the sense of ULIRGs evolution in transition into the classical QSOs. We stress that additional NOEMA CO observations are required to investigate this source in detail, particularly the presence of a possible $\mathrm{CO}$ outflow.

\section{SUMMARY}

In this work, we present the deep NOEMA interferometric observations of $\mathrm{CO}(1-0)$ line and $3 \mathrm{~mm}$ continuum emission in the ultraluminous IR QSO IRAS F07599+6508 at $z=0.1486$. This system shares many physical properties with Mrk 231, the nearest and most luminous IR QSO, in multi-wavelength. Both the $\mathrm{CO}$ line and $3 \mathrm{~mm}$ continuum emission are resolved in our observations, and the $\mathrm{CO}$ gas is found to be spa- 

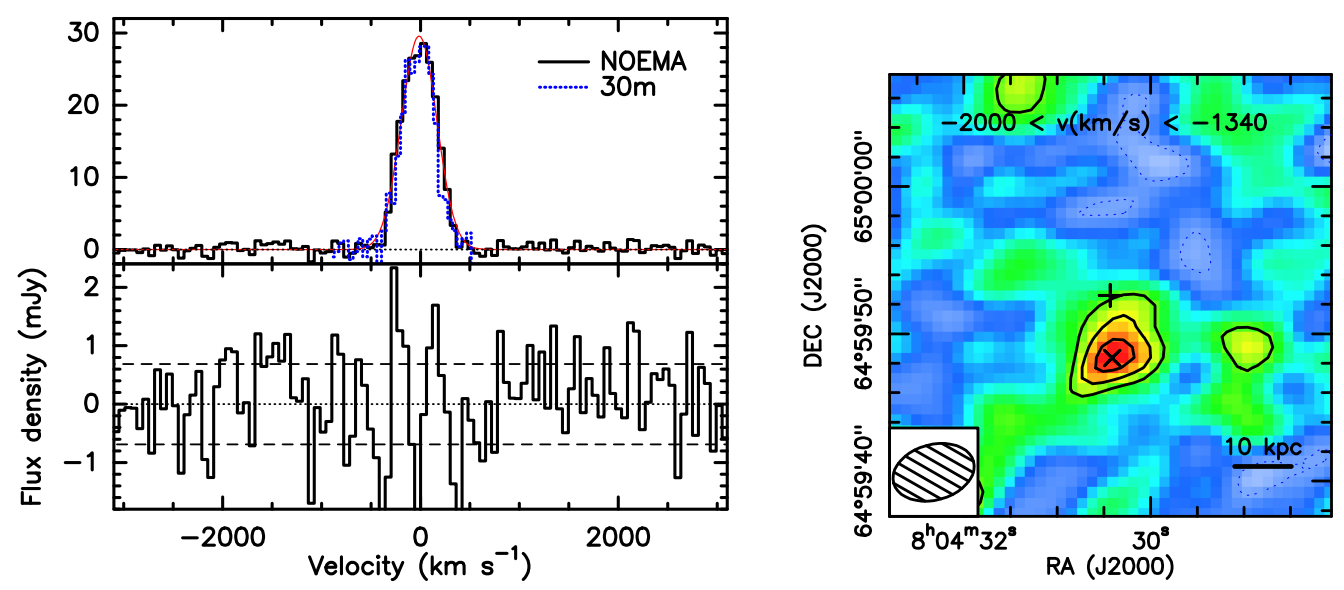

Figure 9. Left: continuum-subtracted spectra of the $\mathrm{CO}(1-0)$ emission line (top) and the residuals (bottom) after removing a Gaussian fit (red line in the top panel) to the CO spectrum of IRAS F07599+6508. In the top panel, the NOEMA CO spectrum (black) is obtained by summing up the flux of the three decomposed components in each channel (see the left panel in Figure 4), while the IRAM 30m CO spectrum (blue) is also plotted for a comparison. In the bottom panel, the dashed lines denote the $1 \sigma$ level. The spectra are binned to a velocity resolution of $\sim 60 \mathrm{~km} \mathrm{~s}^{-1}$. Right: contours from $\mathrm{CO}(1-0)$ dirty maps integrated over the velocity range of $[-1340,-2000] \mathrm{km} \mathrm{s}^{-1}$. Contours starts at $\pm 2 \sigma$ and increase in steps of $1 \sigma\left(81 \mu \mathrm{Jy} \mathrm{beam}{ }^{-1}\right) \mathrm{with}$ positive (negative) contours shown as solid (dotted) lines. The plus sign and cross indicate the CO peak positions of F07599C and F07599S, respectively. The synthesized beam is shown in the bottom-left corner.

tially decomposed into three components. Combining these observations with results from multi-wavelength data (i.e., radio, optical, and infrared), we study the properties of the $3 \mathrm{~mm}$ continuum emission and the $\mathrm{CO}$ components spatially decomposed, and the main results are summarized as follows.

1. The $3 \mathrm{~mm}$ continuum emission is resolved with a deconvolved size of $1 .^{\prime \prime} 5(\sim 4.0 \mathrm{kpc})$. Assuming a nonthermal spectral index of $\alpha^{\mathrm{NT}}=-1.18$, equal to the slope measured between 9.7 and $17.2 \mathrm{GHz}$ representing the steepest part of the radio spectrum, we find that $\sim 30 \%$ of the emission at $3 \mathrm{~mm}$ arises from a free-free component, significantly lower than the fraction $(\sim 70 \%)$ expected for a normal star-forming galaxy. This suggests that the nonthermal synchrotron component from AGN may contribute substantially to the $3 \mathrm{~mm}$ emission. The relatively low FIR-to-radio flux ratio of $q=1.67$ derived also show evidence for an additional contribution from the central AGN that boosting the radio flux. We estimate an SFR of $77 M_{\odot} \mathrm{yr}^{-1}$ based on the thermal free-free emission component at $3 \mathrm{~mm}$, which is close to the IR estimate of $150 M_{\odot} \mathrm{yr}^{-1}$ corrected for the AGN contribution to the bolometric luminosity.

2. Two off-center CO subcomponents, F07599S and F07599NE, located to the south and northeast of the main component F07599C with a projected distance of $\sim 11.4$ and $19.1 \mathrm{kpc}$, respectively, were identified by model fits to the $u v$-data. More evidence for the reality of F07599S and F07599NE are given by the spatial coincidence of the tidal-tail-like feature and the faint peak in the optical images, and the potentially detected clumpy structures in the $1.49 \mathrm{GHz}$ radio image with these two components. The fraction of F07599S and F07599NE contributed to the total CO flux are $9 \%$ and $4 \%$, respectively. Analysis of the CO kinematics together with the multi-wavelengths data for the decomposed components suggests that the gas in the dominant component F07599C is likely rotationally supported with a systematic velocity gradient observed, while F07599S may represent a separate component associated with a merger and F07599NE probably have a different origin.

3. Careful analysis of our current $\mathrm{CO}(1-0)$ data indicates that, the significance of the high-velocity emission feature seen in the blue-shifted side of the spectrum is not sufficient for claiming the detection of a CO outflow in IRAS F07599+6508. It should be noted that, however, the spatially broad CO core distribution with a size larger than $6 \mathrm{kpc}$ is in marked contrast with the compact $\sim$ kpc CO concentration in Mrk 231, where strong molecular outflows were observed over wide velocity ranges.

Additional deeper observations with higher angular resolution are required to better constrain the $\mathrm{CO}$ morphology and kinematics on kpc and sub-kpc scales, and confirm the presence of high-velocity gas and further determine whether the features seen here are potential signs of outflowing gas that is escaping from the host galaxy. 


\section{ACKNOWLEDGMENTS}

We thank the anonymous referee for the constructive comments that helped improve the paper. We acknowledge the support from IRAM staff during the observations and data reduction. This work is based on observations carried out under project number S18CA with the IRAM NOEMA Interferometer. IRAM is supported by INSU/CNRS (France), MPG (Germany) and IGN (Spain).

This work was supported by National Key Basic Research and Development (R\&D) Program of China (Grant No. 2017YFA0402704), NSFC Grant No. 11803090, 11861131007, 12033004, and 11420101002, and Chinese Academy of Sciences Key Research Program of Frontier Sciences (Grant No. QYZDJ-SSWSLH008). X.Y.X and C.N.H. acknowledge support from the NSFC (Grant No. 11733002). The work by K.K is supported by the JSPS KAKENHI Grant Number JP17H06130 and the NAOJ ALMA Scientific Research Grant Number 2017-06B.

Facilities: IRAM:NOEMA, VLA, Sloan, HST, PS1

Software: GILDAS (Guilloteau \& Lucas 2000), CASA (McMullin et al. 2007), AIPS (van Moorsel et al. 1996)

\section{APPENDIX}

\section{A. DECOMPOSITION OF CO EMISSION}

To illustrate the spatial decomposition of CO emission in IRAS F07599+6508, we here describe the process of model fitting procedure. We fit the model to the $u v$-data using combinations of functions by allowing all parameters to be free. Combinations of models in a successive progression of complexity started with a point source and then circular Gaussian, and elliptical Gaussian. The simultaneous fit was rerun each time as necessary to achieve a uniform residual (i.e., no significant peak or negative regions $\gtrsim 3 \sigma$ ) after subtraction of the models. By verifying the evolution of the uncertainties on the fitted parameters, we found that the best fit was obtained with a combination of an elliptical Gaussian (F07599C) and two point sources (F07599S and F07599NE) functions with minimum uncertainties on the fitted parameters. The residuals after subtracting an elliptical Gaussian plus two point source components are shown in the right panel of Figure 3. It should be noted that there are a few peaks with SNR 3 (e.g., the peaks to the northwest of F07599S and F07599NE, respectively) in the residual map, indicative of the likely presence of extended structure. These weak features are however difficult to be tested with the current data. We should stress that higher spatial resolution observations are needed to confirm these findings.

\section{B. CHANNEL MAPS OF CO(1-0) EMISSION}

The spatial and velocity distribution of $\mathrm{CO}(1-0)$ emission in IRAS F07599+6508 can be further explored by inspecting the channel maps in Figure 10, which shows the image of CO emission within an interval of $\sim 120 \mathrm{~km} \mathrm{~s}^{-1}$ encompassing the velocity range from $-2090 \mathrm{~km} \mathrm{~s}^{-1}$ to $2093 \mathrm{~km} \mathrm{~s}^{-1}$. The channel maps were produced from the continuum-subtracted data cube that was cleaned down to the $1 \sigma$ noise level. The extended structures to the south and northeast of galaxy center observed in each channel map, spatially coincident with the subcomponent of F07599S and F07599NE (marked as red crosses in Figure 10), show evidence for the reality of such features.

\section{RADIO MAPS AND FLUX MEASUREMENTS}

Table 2 summarizes the radio continuum properties of IRAS F07599+6508 obtained with VLA. Except the 22.46 $\mathrm{GHz}$ data that was obtained in a single $\mathrm{CD}$ configuration, observations at the rest of frequencies were made with multiple arrays.

To investigate the distribution of radio emission and make a comparison with $\mathrm{CO}$ emission, we show the map obtained with higher angular resolution for each frequency in Figure 11. For 14.94 and $22.46 \mathrm{GHz}$, the emission are detected with a compact morphology, while for the emission at 4.86 and $8.44 \mathrm{GHz}$ where the observations were deeper, the radio source exhibits more complex morphology with clumpy structures outside the main body of the galaxy to the east and west, respectively. The peak flux density measured in different configuration were found to be in good agreement for each frequency (i.e., to within $\sim 10 \%$; see Table 2). The highest resolution image was obtained at $17.2 \mathrm{GHz}$ with beam size of $\sim 0 . " 18(\sim 0.47 \mathrm{kpc})$, of which the peak flux density measured is found to be comparable with that measured from CD configuration (beam size $\sim 4 .{ }^{\prime \prime} 58$ ). The consistent peak flux densities measured between different beams for the different bands indicate that the bulk of radio emission probably originates in the inner compact region of the AGN. 


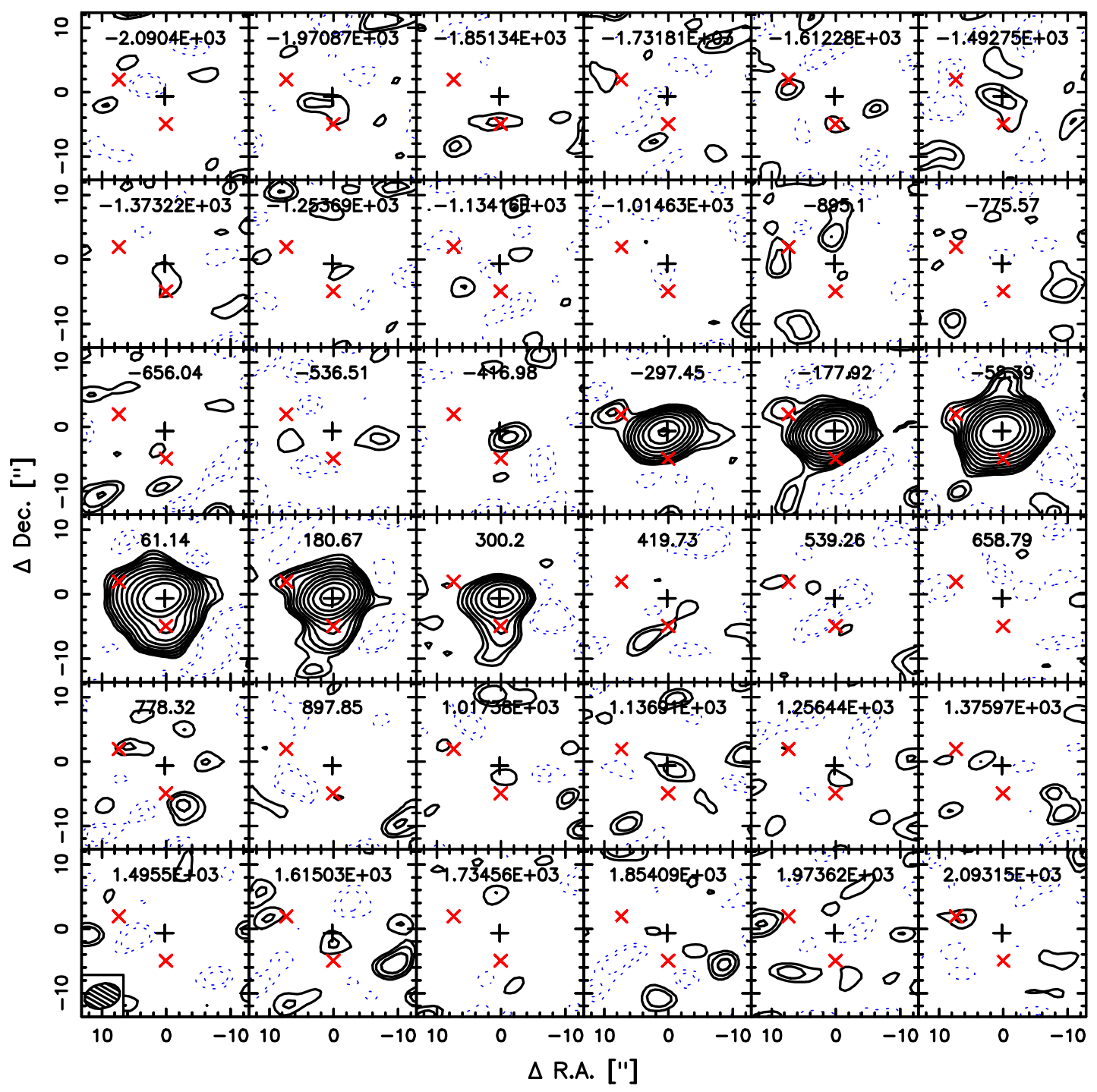

Figure 10. Channel maps showing the CO(1-0) emission of IRAS F07599+6508 in the $[-2000,+1700] \mathrm{km} \mathrm{s}^{-1} \mathrm{range}^{\mathrm{with}}$ channel wide of $\sim 120 \mathrm{~km} \mathrm{~s}^{-1}$. The red crosses mark the positions of CO subcomponents (F07599S and F07599NE) and the black plus sign indicates the peak of $3 \mathrm{~mm}$ continuum emission. Contour levels start at $\pm 1.5 \sigma$ and increase by factors of 1.5 with positive (negative) contours shown as solid (blue dotted) lines. The velocities in $\mathrm{km} \mathrm{s}^{-1}$ are marked in the top left corner of each channel map and the synthesized beam is shown in the bottom-left corner.

\section{MULTI-WAVELENGTH DATA OF IRAS F07599+6508 AND A COMPANION SOURCE TO THE NORTHWEST}

In this appendix we show the multi-wavelength (from GALEX near-UV to WISE $3.4 \mu \mathrm{m}$ band) data of IRAS F07599+6508 and a companion source WISEA J080428.28+650001.7 to the northwest (Figure 12). J0804+6500 has a distance of $17 .^{\prime \prime} 5(\sim 45 \mathrm{kpc})$ from IRAS F07599+6508, with a coordinates of 08:04:28.140, +65:00:02.25.

Figure 13 shows the NOEMA spectra (without correction for primary beam attenuation (PBA)) extracted from the position of J0804+6500 by fitting a point-source model to the uv-data. The tuning frequency covered in the upper and lower sidebands corresponds to a redshift range of 0.128 to 0.223 and 0.238 to 0.462 , respectively, if associated with a $\mathrm{CO}(1-0)$ transition. The $1 \sigma \mathrm{rms}$ noise level of the spectrum in the USB and LSB shown in Figure 13 is 0.20 mJy and 0.21 mJy, respectively. We do not detect any significant emission from J0804+6500 in our observations.

\section{REFERENCES}

Aalto, S., Garcia-Burillo, S., Muller, S., et al. 2012, A\&A, 537, A44, doi: 10.1051/0004-6361/201117919
Barcos-Muñoz, L., Leroy, A. K., Evans, A. S., et al. 2017, ApJ, 843, 117, doi: 10.3847/1538-4357/aa789a 

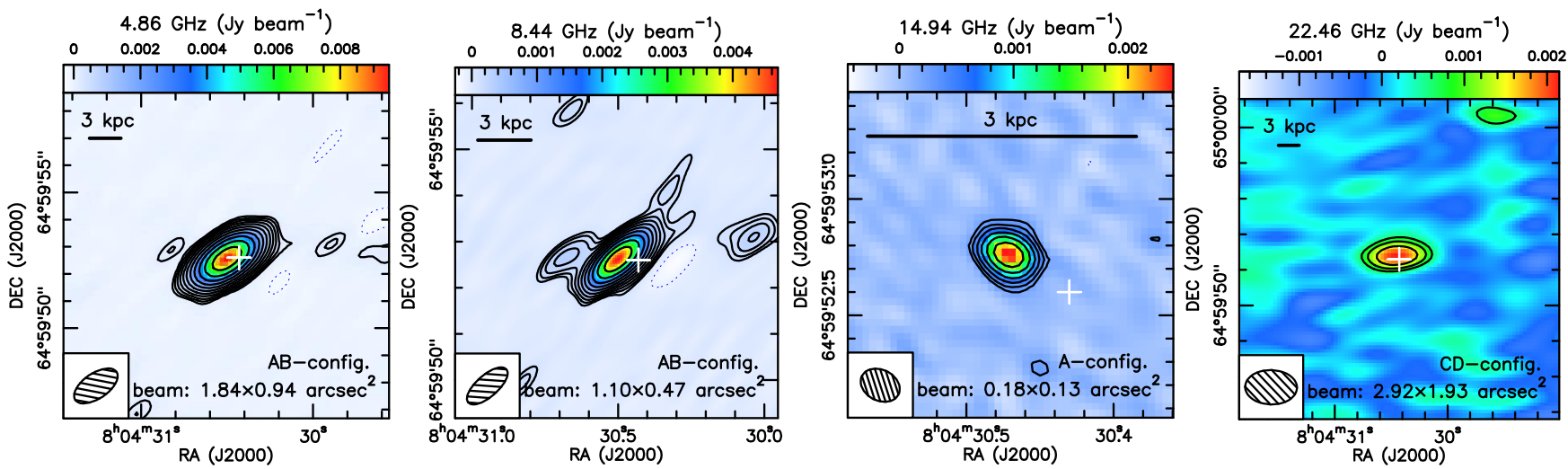

Figure 11. VLA multi-band radio continuum imaging of IRAS F07599+6508 at 4.86, 8.44, 14.94, and 22.46 GHz from left to right. Except the observations at $22.46 \mathrm{GHz}$ which was conducted in a single array of CD configuration, the rest of observations were made with arrays in both AB configuration (A configuration for $14.94 \mathrm{GHz}$ ) and CD configuration. Here we only show the maps with higher angular resolution. All the contours start at $3 \sigma$ and increase by a factor of 1.5 . The synthesized beam and beam size of each radio observations are shown in the bottom of each panel. The white plus sign indicates the peak of 3 mm continuum emission.

Table 2. VLA radio continuum properties of IRAS F07599+6508

\begin{tabular}{|c|c|c|c|c|c|}
\hline $\begin{array}{l}\text { Obs. freq. } \\
(\mathrm{GHz})\end{array}$ & $\begin{array}{c}\mathrm{RA}^{a} \\
(\mathrm{~J} 2000)\end{array}$ & $\begin{array}{l}\mathrm{DEC}^{a} \\
(\mathrm{~J} 2000)\end{array}$ & Conf. & $\begin{array}{l}\text { Synt.beam } \\
(\operatorname{arcsec})\end{array}$ & $\begin{array}{c}\mathrm{S}_{\text {peak }} \\
\left(\mathrm{mJy}_{\text {beam }}{ }^{-1}\right)\end{array}$ \\
\hline \multirow[t]{2}{*}{$1.49 \mathrm{GHz}$} & 08:04:30.53 & $64: 59: 53.3$ & $\mathrm{AB}$ & $5.33 \times 2.08$ & $32.33 \pm 0.10$ \\
\hline & & & $\mathrm{CD}$ & $65.2 \times 28.3$ & $36.72 \pm 0.16$ \\
\hline \multirow[t]{2}{*}{$4.86 \mathrm{GHz}$} & 08:04:30.51 & $64: 59: 52.6$ & $\mathrm{AB}$ & $1.84 \times 0.94$ & $9.59 \pm 0.05$ \\
\hline & & & CD & $20.8 \times 8.8$ & $9.15 \pm 0.16$ \\
\hline \multirow[t]{2}{*}{$8.44 \mathrm{GHz}$} & 08:04:30.51 & 64:59:52.6 & $\mathrm{AB}$ & $1.10 \times 0.47$ & $4.67 \pm 0.07$ \\
\hline & & & $\mathrm{CD}$ & $11.0 \times 5.1$ & $5.23 \pm 0.09$ \\
\hline \multirow[t]{2}{*}{$14.94 \mathrm{GHz}$} & 08:04:30.471 & $64: 59: 52.76$ & $\mathrm{~A}$ & $0.18 \times 0.13$ & $2.38 \pm 0.06$ \\
\hline & & & $\mathrm{CD}$ & $4.58 \times 2.56$ & $2.69 \pm 0.08$ \\
\hline $22.46 \mathrm{GHz}$ & 08:04:30.46 & $64: 59: 52.8$ & $\mathrm{CD}$ & $2.92 \times 1.93$ & $2.20 \pm 0.10$ \\
\hline
\end{tabular}

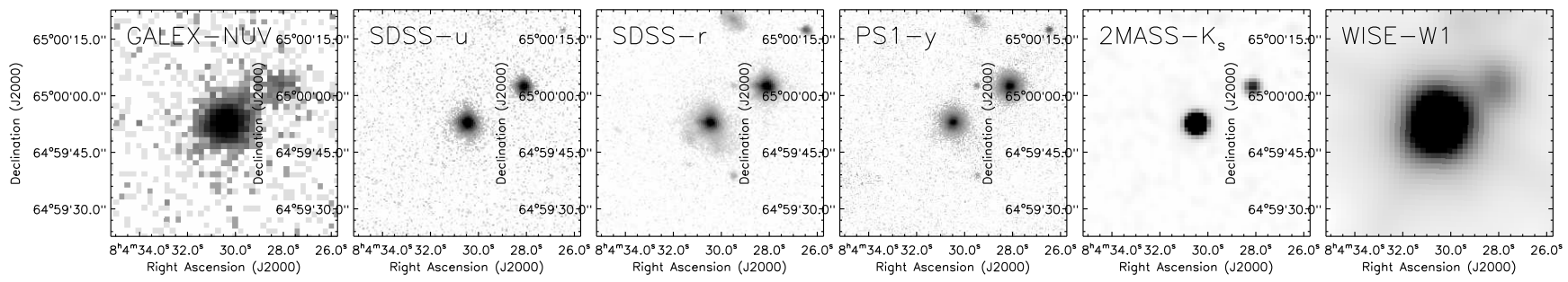

Figure 12. Image cutouts $\left(60^{\prime \prime} \times 60^{\prime \prime}\right.$ on a side) of IRAS F07599+6508 and a companion source WISEA J080428.28+650001.7 to the northwest. From left to right, we show the GALEX near-UV, SDSS $u-, r-$, Pan-STARRS1 $y$-, 2 MASS K $\mathrm{s}^{-}$, and WISE $3.4 \mu \mathrm{m}$-band images. 

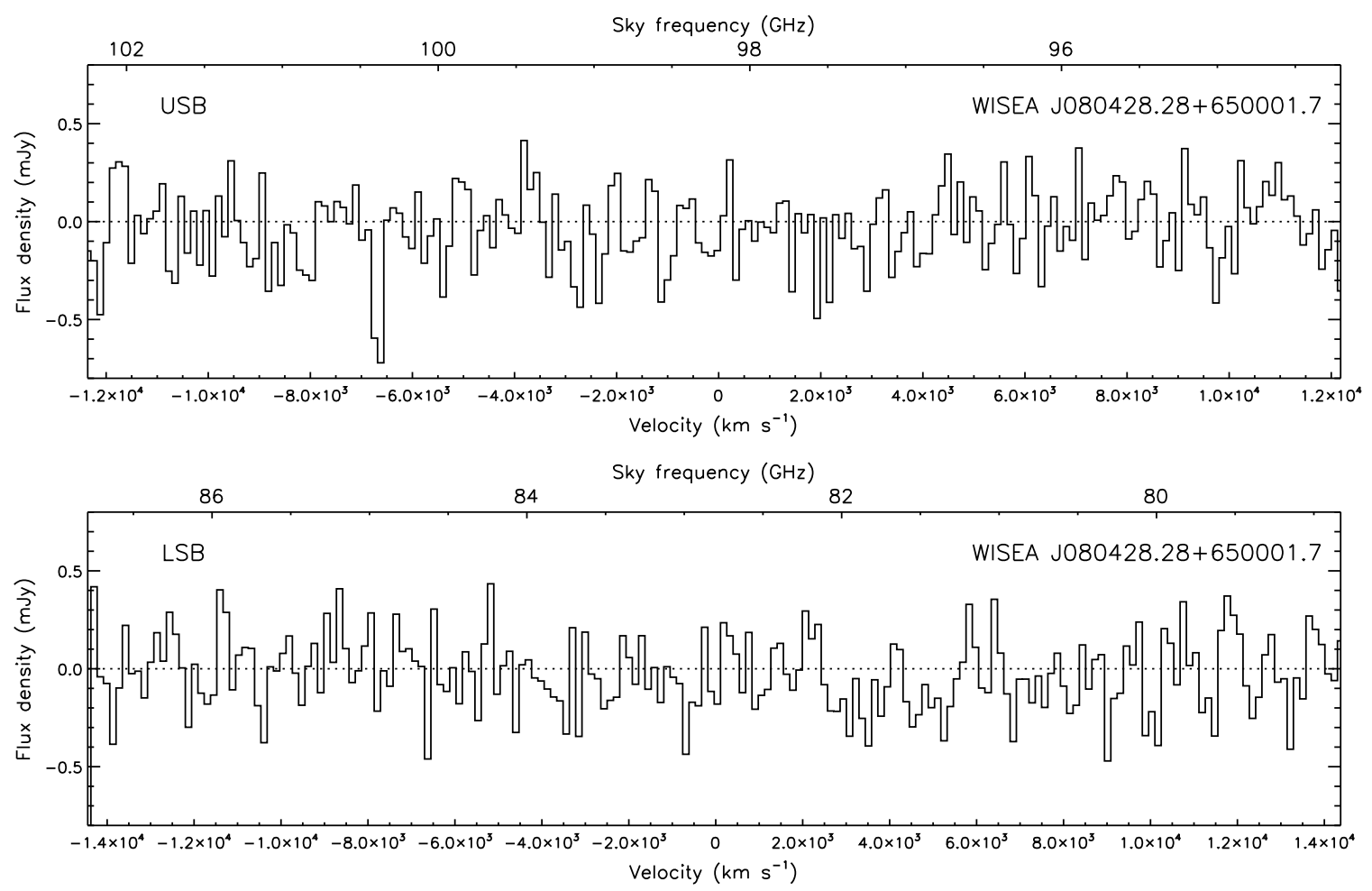

Figure 13. NOEMA spectrum (without correction for PBA) of WISEA J080428.28+650001.7 covered in the USB (top) and the LSB (bottom). The spectra are extracted by fitting a point-source model to the $u v$-data and are binned to a channel width of $\sim 120-140 \mathrm{~km} \mathrm{~s}^{-1}$. 
Barnes, J. E., \& Hernquist, L. 1992, ARA\&A, 30, 705, doi: 10.1146/annurev.aa.30.090192.003421

Behar, E., Baldi, R. D., Laor, A., et al. 2015, MNRAS, 451, 517, doi: 10.1093/mnras/stv988

Bolatto, A. D., Wolfire, M., \& Leroy, A. K. 2013, ARA\&A, 51, 207, doi: 10.1146/annurev-astro-082812-140944

Boroson, T. A., \& Meyers, K. A. 1992, ApJ, 397, 442, doi: $10.1086 / 171800$

Brightman, M., \& Nandra, K. 2011, MNRAS, 414, 3084, doi: 10.1111/j.1365-2966.2011.18612.x

Bryant, P. M., \& Scoville, N. Z. 1996, ApJ, 457, 678, doi: 10.1086/176763

Canalizo, G., \& Stockton, A. 2001, ApJ, 555, 719, doi: $10.1086 / 321520$

Cao, C., Xia, X. Y., Wu, H., et al. 2008, MNRAS, 390, 336, doi: 10.1111/j.1365-2966.2008.13747.x

Cicone, C., Feruglio, C., Maiolino, R., et al. 2012, A\&A, 543, A99, doi: 10.1051/0004-6361/201218793

Cicone, C., Maiolino, R., Aalto, S., Muller, S., \& Feruglio, C. 2020, A\&A, 633, A163, doi: 10.1051/0004-6361/201936800

Cicone, C., Maiolino, R., Sturm, E., et al. 2014, A\&A, 562, A21, doi: 10.1051/0004-6361/201322464

Cicone, C., Maiolino, R., Gallerani, S., et al. 2015, A\&A, 574, A14, doi: 10.1051/0004-6361/201424980

Cicone, C., Severgnini, P., Papadopoulos, P. P., et al. 2018, ApJ, 863, 143, doi: 10.3847/1538-4357/aad32a

Clemens, M. S., Scaife, A., Vega, O., \& Bressan, A. 2010, MNRAS, 405, 887, doi: 10.1111/j.1365-2966.2010.16534.x

Clemens, M. S., Vega, O., Bressan, A., et al. 2008, A\&A, 477, 95, doi: 10.1051/0004-6361:20077224

Clements, D. L., Pearson, C., Farrah, D., et al. 2018, MNRAS, 475, 2097, doi: 10.1093/mnras/stx3227

Condon, J. J. 1992, ARA\&A, 30, 575, doi: 10.1146/annurev.aa.30.090192.003043

Condon, J. J., Cotton, W. D., Greisen, E. W., et al. 1998, AJ, 115, 1693, doi: 10.1086/300337

Daddi, E., Bournaud, F., Walter, F., et al. 2010, ApJ, 713, 686, doi: 10.1088/0004-637X/713/1/686

Davies, R., Förster Schreiber, N. M., Cresci, G., et al. 2011, ApJ, 741, 69, doi: 10.1088/0004-637X/741/2/69

Di Matteo, T., Springel, V., \& Hernquist, L. 2005, Nature, 433, 604, doi: 10.1038/nature03335

Downes, D., \& Solomon, P. M. 1998, ApJ, 507, 615, doi: 10.1086/306339

Evans, A. S., Frayer, D. T., Surace, J. A., \& Sanders, D. B. 2001, AJ, 121, 1893, doi: 10.1086/319972

Evans, A. S., Solomon, P. M., Tacconi, L. J., Vavilkin, T., \& Downes, D. 2006, AJ, 132, 2398, doi: 10.1086/508416
Fabian, A. C. 2012, ARA\&A, 50, 455, doi: 10.1146/annurev-astro-081811-125521

Feruglio, C., Maiolino, R., Piconcelli, E., et al. 2010, A\&A, 518, L155, doi: 10.1051/0004-6361/201015164

Feruglio, C., Fiore, F., Carniani, S., et al. 2015, A\&A, 583, A99, doi: 10.1051/0004-6361/201526020

Fischer, J., Sturm, E., González-Alfonso, E., et al. 2010, A\&A, 518, L41, doi: 10.1051/0004-6361/201014676

Greve, T. R., Bertoldi, F., Smail, I., et al. 2005, MNRAS, 359, 1165, doi: 10.1111/j.1365-2966.2005.08979.x

Guilloteau, S., \& Lucas, R. 2000, in Astronomical Society of the Pacific Conference Series, Vol. 217, Imaging at Radio through Submillimeter Wavelengths, ed. J. G. Mangum \& S. J. E. Radford, 299

Hao, C. N., Xia, X. Y., Mao, S., Wu, H., \& Deng, Z. G. 2005, ApJ, 625, 78, doi: 10.1086/429716

Hao, C.-N., Xia, X.-Y., Shu-DeMao, Deng, Z.-G., \& Wu, H. 2008, ChJA\&A, 8, 12, doi: 10.1088/1009-9271/8/1/02

Ho, L. C. 2007, ApJ, 668, 94, doi: 10.1086/521270

Hopkins, P. F., Hernquist, L., Cox, T. J., et al. 2006, ApJS, 163, 1, doi: 10.1086/499298

Hopkins, P. F., Hernquist, L., Cox, T. J., \& Kereš, D. 2008, ApJS, 175, 356, doi: 10.1086/524362

Imanishi, M., Nakanishi, K., \& Izumi, T. 2019, ApJS, 241, 19, doi: 10.3847/1538-4365/ab05b9

Inoue, Y., \& Doi, A. 2014, PASJ, 66, L8, doi: $10.1093 / \mathrm{pasj} / \mathrm{psu} 079$

—. 2018, ApJ, 869, 114, doi: 10.3847/1538-4357/aaeb95

Jarvis, M. E., Harrison, C. M., Mainieri, V., et al. 2020, MNRAS, 498, 1560, doi: 10.1093/mnras/staa2196

Kim, D. C., \& Sanders, D. B. 1998, ApJS, 119, 41, doi: $10.1086 / 313148$

King, A., \& Pounds, K. 2015, ARA\&A, 53, 115, doi: 10.1146/annurev-astro-082214-122316

Kormendy, J., \& Ho, L. C. 2013, ARA\&A, 51, 511, doi: 10.1146/annurev-astro-082708-101811

Kwan, J., Cheng, F.-Z., Fang, L.-Z., Zheng, W., \& Ge, J. 1995, ApJ, 440, 628, doi: 10.1086/175302

La Caria, M. M., Vignali, C., Lanzuisi, G., Gruppioni, C., \& Pozzi, F. 2019, MNRAS, 487, 1662, doi: $10.1093 / \mathrm{mnras} / \mathrm{stz} 1381$

Laor, A., \& Behar, E. 2008, MNRAS, 390, 847, doi: 10.1111/j.1365-2966.2008.13806.x

Lawrence, A., Rowan-Robinson, M., Ellis, R. S., et al. 1999, MNRAS, 308, 897, doi: 10.1046/j.1365-8711.1999.02593.x

Le Floc'h, E., Papovich, C., Dole, H., et al. 2005, ApJ, 632, 169, doi: 10.1086/432789

Lonsdale, C. J., Lonsdale, C. J., Smith, H. E., \& Diamond, P. J. 2003, ApJ, 592, 804, doi: 10.1086/375778 
Luo, B., Brandt, W. N., Alexander, D. M., et al. 2014, ApJ, 794, 70, doi: 10.1088/0004-637X/794/1/70

McMullin, J. P., Waters, B., Schiebel, D., Young, W., \& Golap, K. 2007, in Astronomical Society of the Pacific Conference Series, Vol. 376, Astronomical Data Analysis Software and Systems XVI, ed. R. A. Shaw, F. Hill, \& D. J. Bell, 127

Murphy, E. J., Bremseth, J., Mason, B. S., et al. 2012, ApJ, 761, 97, doi: 10.1088/0004-637X/761/2/97

Neeleman, M., Novak, M., Venemans, B. P., et al. 2021, arXiv e-prints, arXiv:2102.05679.

https://arxiv.org/abs/2102.05679

Panessa, F., Baldi, R. D., Laor, A., et al. 2019, Nature Astronomy, 3, 387, doi: 10.1038/s41550-019-0765-4

Raginski, I., \& Laor, A. 2016, MNRAS, 459, 2082, doi: 10.1093/mnras/stw772

Rupke, D. S. N., Gültekin, K., \& Veilleux, S. 2017, ApJ, 850, 40, doi: 10.3847/1538-4357/aa94d1

Rupke, D. S. N., \& Veilleux, S. 2011, ApJL, 729, L27, doi: 10.1088/2041-8205/729/2/L27

Sanders, D. B., \& Mirabel, I. F. 1996, ARA\&A, 34, 749, doi: 10.1146/annurev.astro.34.1.749

Sanders, D. B., Soifer, B. T., Elias, J. H., et al. 1988a, ApJ, 325, 74, doi: 10.1086/165983

Sanders, D. B., Soifer, B. T., Elias, J. H., Neugebauer, G., \& Matthews, K. 1988b, ApJL, 328, L35, doi: $10.1086 / 185155$

Scoville, N. Z., Frayer, D. T., Schinnerer, E., \& Christopher, M. 2003, ApJL, 585, L105, doi: 10.1086/374544

Shangguan, J., Ho, L. C., Bauer, F. E., Wang, R., \& Treister, E. 2020, ApJS, 247, 15, doi: 10.3847/1538-4365/ab5db2

Silk, J., \& Rees, M. J. 1998, A\&A, 331, L1. https://arxiv.org/abs/astro-ph/9801013

Soifer, B. T., Sanders, D. B., Madore, B. F., et al. 1987, ApJ, 320, 238, doi: 10.1086/165536

Solomon, P. M., Downes, D., Radford, S. J. E., \& Barrett, J. W. 1997, ApJ, 478, 144, doi: 10.1086/303765

Spoon, H. W. W., Farrah, D., Lebouteiller, V., et al. 2013, ApJ, 775, 127, doi: 10.1088/0004-637X/775/2/127
Surace, J. A., \& Sanders, D. B. 2000, AJ, 120, 604, doi: $10.1086 / 301491$

Tadaki, K.-i., Iono, D., Yun, M. S., et al. 2020, ApJ, 889, 141, doi: 10.3847/1538-4357/ab64f4

Tan, Q.-H., Gao, Y., Kohno, K., et al. 2019, ApJ, 887, 24, doi: 10.3847/1538-4357/ab50be

Taylor, G. B., Silver, C. S., Ulvestad, J. S., \& Carilli, C. L. 1999, ApJ, 519, 185, doi: 10.1086/307362

Ueda, J., Iono, D., Yun, M. S., et al. 2014, ApJS, 214, 1, doi: 10.1088/0067-0049/214/1/1

van Moorsel, G., Kemball, A., \& Greisen, E. 1996, in Astronomical Society of the Pacific Conference Series, Vol. 101, Astronomical Data Analysis Software and Systems V, ed. G. H. Jacoby \& J. Barnes, 37

Vega, O., Clemens, M. S., Bressan, A., et al. 2008, A\&A, 484, 631, doi: 10.1051/0004-6361:20078883

Veilleux, S., Kim, D. C., \& Sanders, D. B. 1999, ApJ, 522, 113, doi: 10.1086/307634

Veilleux, S., Rupke, D. S. N., Kim, D. C., et al. 2009, ApJS, 182, 628, doi: 10.1088/0067-0049/182/2/628

Veilleux, S., Meléndez, M., Sturm, E., et al. 2013, ApJ, 776, 27, doi: 10.1088/0004-637X/776/1/27

Véron-Cetty, M. P., Joly, M., Véron, P., et al. 2006, A\&A, 451, 851, doi: 10.1051/0004-6361:20054237

Wang, J., Li, D., Goldsmith, P. F., et al. 2020, ApJ, 889, 129, doi: 10.3847/1538-4357/ab612d

Wang, R., Carilli, C. L., Neri, R., et al. 2010, ApJ, 714, 699, doi: 10.1088/0004-637X/714/1/699

Wilson, C. D., Petitpas, G. R., Iono, D., et al. 2008, ApJS, 178, 189, doi: 10.1086/590910

Xia, X.-Y., Boller, T., Deng, Z.-G., \& Börner, G. 2001, ChJA\&A, 1, 221, doi: 10.1088/1009-9271/1/3/221

Xia, X. Y., Gao, Y., Hao, C.-N., et al. 2012, ApJ, 750, 92, doi: 10.1088/0004-637X/750/2/92

Xu, C. K., Cao, C., Lu, N., et al. 2014, ApJ, 787, 48, doi: 10.1088/0004-637X/787/1/48

—. 2015, ApJ, 799, 11, doi: 10.1088/0004-637X/799/1/11

Yun, M. S., Reddy, N. A., \& Condon, J. J. 2001, ApJ, 554, 803, doi: 10.1086/323145

Zheng, X. Z., Xia, X. Y., Mao, S., Wu, H., \& Deng, Z. G. 2002, AJ, 124, 18, doi: 10.1086/340964 